This item was submitted to Loughborough's Research Repository by the author.

Items in Figshare are protected by copyright, with all rights reserved, unless otherwise indicated.

\title{
Variable and constant returns-to-scale production technologies with component processes
}

PLEASE CITE THE PUBLISHED VERSION

https://doi.org/10.1287/opre.2021.2103

PUBLISHER

INFORMS

VERSION

AM (Accepted Manuscript)

\section{PUBLISHER STATEMENT}

This paper was accepted for publication in the journal Operations Research and the definitive published version is available at https://doi.org/10.1287/opre.2021.2103.

\section{LICENCE}

CC BY-NC-ND 4.0

\section{REPOSITORY RECORD}

Podinovski, Victor. 2021. "Variable and Constant Returns-to-scale Production Technologies with Component Processes". Loughborough University. https://hdl.handle.net/2134/13011254.v1. 


\title{
Submitted to Operations Research manuscript (Please, provide the mansucript number!)
}

\section{Variable and Constant Returns-to-Scale Production Technologies with Component Processes}

\author{
Victor V. Podinovski \\ School of Business and Economics, Loughborough University, Loughborough LE11 3TU, United Kingdom, \\ v.podinovski@lboro.ac.uk
}

\begin{abstract}
We consider nonparametric production technologies characterized by several component production processes, and allow both component-specific and shared inputs and outputs. Each process uses its specific inputs and an unknown part of the shared inputs, to produce its specific outputs and an unknown part of the shared outputs. For the described setting, we develop two new models of production technologies, under the assumptions of variable and constant returns to scale (VRS and CRS). These models are based on the worst-case assumption about the allocation of the shared inputs and outputs to component processes and, therefore, do not require exact knowledge of the actual allocation. The new models are larger than the standard VRS and CRS technologies. We provide a formal axiomatic derivation of the new technologies, explore their dual interpretation and demonstrate their usefulness in an application.
\end{abstract}

Key words: data envelopment analysis; efficiency; convexity; multiple-component technology

\section{Introduction}

Data envelopment analysis (DEA) is an established nonparametric methodology for measuring the efficiency of organizational units, referred to as decision making units (DMUs). The two conventional DEA models are based on the constant and variable returns-to-scale (CRS and VRS) production technologies. These technologies were introduced to the DEA literature by the pioneering works of Charnes et al. (1978) and Banker et al. (1984), while their earlier statements can also be found in the economics literature (see, e.g., Afriat 1972, Shephard 1974 and Färe et al. 1983).

Both the CRS and VRS models of technology implicitly assume that all inputs are used in the production of all outputs. However, it has long been realised that this assumption may often be too simplistic and generic. Indeed, there are many examples in which the technology may be regarded as a combination of several component production processes. For example, in the efficiency assessment 
of universities, Beasley (1995) identifies the teaching and research component processes that have component-specific inputs and outputs, and also shared inputs. In the context of school assessment, Podinovski et al. (2018) consider the teaching of the academic, vocational and alternative programs as the three component processes with a number of component-specific and shared inputs and outputs. For bank branches, Cook et al. (2000) and Cook and Zhu (2006, 2011) show that the sales of financial products and counter transactions may be viewed as component processes with both specific and shared types of input. In the context of manufacturing plants, examples of multicomponent technologies are explored by Cook and Green (2004), Cook et al. (2013) and Imanirad et al. (2013). Various technologies with component processes are also considered in the literature on network DEA where they are often referred to as parallel structures - see a review in Kao (2014).

The standard VRS and CRS technologies are generally too small for the purpose of modeling multiple-component production processes. Indeed, if we recognise the distinct nature of each component, then we should have more freedom in combining and scaling such components irrespective of the other components, compared to the standard VRS and CRS models in which such combinations are allowed only with respect to whole DMUs.

The idea of treating individual component processes independently of the other processes has been explored in the literature. The main difficulty here is that we do not generally know in what proportions the shared inputs and outputs should be attributed to each component process. For example, the administration costs and other expenditures of an organization are often reported as an overall figure. This is treated as a shared input whose exact allocation to different component processes is unknown. Similarly, in education applications, the overall numbers of students with good exam results may be treated as a shared output whose allocation to different programs of study is unavailable.

The literature on this subject suggests only a few conceptually different ideas as to how the shared inputs and outputs can be allocated to different component processes, and all of them have important limitations. 
Podinovski: Technologies with Multiple Component Processes

Cook et al. (2000) and Cook and Green (2004) suggest a variant of the multiplier CRS model stated in terms of the input and output weights which reflects the multi-component structure of the production process. The main idea of this approach is that the allocation of the shared inputs is variable and, at an optimal solution, is the most favorable for the DMU under the assessment. A similar idea for the multiplier VRS model is explored by Cook and Hababou (2001), Cook and Zhu (2011) and Ding et al. (2015). It is worth noting that this approach is developed entirely in the multiplier framework. The dual envelopment model in this case turns out excessively complex and does not allow a simple interpretation. As a consequence, the economic meaning and properties of the underlying technology implicitly used in the multiplier model are unclear. This approach is also based on several apparently heuristic assumptions, e.g., that the variable proportions in which the shared inputs are allocated to a particular production process should be identical for all observed DMUs - see Section 6.2 for further discussion.

Cherchye et al. (2013) and subsequent works (see, e.g., Cherchye et al. 2016 and Walheer 2018) consider VRS and CRS production technologies in which the inputs common to all component production processes are not allocated but are used jointly. The use of any such joint inputs by any DMU does not have a negative effect on the availability of these inputs to the other DMUs. As shown by Cherchye et al. (2013), the incorporation of joint inputs alongside component-specific inputs and outputs is relatively unproblematic. It is, however, clear that the notion of joint inputs is conceptually inappropriate for the shared inputs (and similarly for outputs) that are actually allocated, although in some unknown proportions, to different component processes.

The closest to our paper is the different idea employed by Podinovski et al. (2018) who use the worst-case assumption concerning the allocation of the shared inputs and outputs. This approach is applicable to the modeling of scalable (i.e., CRS) technologies in which different component processes may be scaled with generally different factors. Namely, all component-specific inputs and outputs of each process can be scaled by some nonnegative multiplier. At the same time, all shared inputs are scaled by the maximum of such multipliers, and all shared outputs are scaled by their minimum, taken across all component processes. This treatment is valid for any allocation of the 
shared inputs and outputs to different component processes and does not require the knowledge of the exact allocation.

The approach of Podinovski et al. (2018) utilizes the standard assumption of convexity, according to which only whole DMUs may enter convex combinations, and does not allow such combinations within each component process independently of the other components. It appears that a new idea is needed to allow such component-specific property of convexity. This would allow us to construct a VRS technology in which different component processes could form convex combinations independently of each other. We could further combine this with the idea of Podinovski et al. (2018) of independent component scaling, in order to obtain a multi-component CRS model of technology.

An immediate problem in developing the new notion of convexity for multi-component processes is the treatment of the shared inputs and outputs. In a way conceptually similar to the approach of Podinovski et al. (2018) but different in its actual implementation, in this paper we overcome this problem by considering the worst-case allocation of such inputs and outputs in the construction of convex combinations for each component process. This results in a new statement of the axiom of convexity for multi-component technologies.

The main contribution of this paper is a full axiomatic development of the VRS and CRS technologies with multiple component processes, referred to as the MVRS and MCRS technologies, respectively. Importantly, these new technologies do not require knowledge of the exact allocation of the shared inputs and outputs to the component processes, and do not rely on any particular mechanism of such allocation.

We further explore the dual multiplier models based on the MVRS and MCRS technologies. We show that, similar to the approach explored by Cook et al. (2000) and related subsequent works, the multiplier MVRS and MCRS models incorporate variable proportions that allocate the shared inputs and outputs to component processes in a way that is the most favorable for the DMU under the assessment. However, we show that this allocation mechanism (which is not postulated in our paper but is a formal consequence of the assumed production axioms) is significantly different from the approach of Cook et al. (2000). 
Podinovski: Technologies with Multiple Component Processes

Article submitted to Operations Research; manuscript no. (Please, provide the mansucript number!)

We subsequently turn to the investigation of various scale characteristics of the new MVRS technology. We show that the notions of scale efficiency and returns to scale arising in this technology are natural generalizations of the corresponding conventional scale characteristics of the standard VRS technology, and develop methods of their evaluation.

The new MVRS and MCRS technologies include the corresponding conventional VRS and CRS technologies as subsets and provide a better discrimination on efficiency than the latter. We demonstrate the usefulness of the MVRS and MCRS models by an application to universities in England whose science, non-science and medical departments are considered as component processes.

\section{Preliminaries}

Starting with the conventional setting, let $T \subset \mathbb{R}_{+}^{m+s}$ be a production technology with the inputs $i \in \mathrm{I}=\{1, \ldots, m\}$ and outputs $r \in \mathrm{O}=\{1, \ldots, s\}$. DMUs are elements $(X, Y) \in T$, where $X \in \mathbb{R}_{+}^{m}$ and $Y \in \mathbb{R}_{+}^{s}$ are the vectors of inputs and outputs, respectively. Observed DMUs are denoted $\left(X_{j}, Y_{j}\right), j \in J=\{1, \ldots, n\}$. It is assumed that $X_{j} \neq 0$ and $Y_{j} \neq 0$, for all $j \in J$. Notation $\mathrm{DMU}_{o}$ or $\left(X_{o}, Y_{o}\right)$ is used for the DMU whose efficiency is being evaluated.

In the axiomatic framework, technology $T$ is defined by the set of assumed production axioms (see, e.g., Färe et al. 1985, 1994). Utilizing the minimum extrapolation principle, Banker et al. (1984) show that the standard VRS technology is defined by the following three axioms:

Axiom 1 (Feasibility of Observed DMUs). $\left(X_{j}, Y_{j}\right) \in T$ for any $j \in J$.

Axiom 2 (Free Disposability). If $(X, Y) \in T, Y \geq Y^{\prime} \geq 0$ and $X \leq X^{\prime}$ then $\left(X^{\prime}, Y^{\prime}\right) \in T$.

Axiom 3 (Convexity). Technology $T$ is a convex set.

As also shown by Banker et al. (1984), the CRS technology of Charnes et al. (1978) is defined by Axioms 1-3 and the additional axiom that allows proportional scaling of DMUs:

Axiom 4 (Scalability). If $(X, Y) \in T$ and $\rho \geq 0$ then $(\rho X, \rho Y) \in T$.

Let us now introduce additional notation that reflects the multi-component nature of the production technology. Suppose that there are $K$ component processes. Because the case $K=1$ corresponds to the conventional VRS and CRS technologies, in all our discussions we implicitly assume 
that $K \geq 2$, although our results also remain trivially true for $K=1$.

Consider the following decomposition of the sets I and O into the mutually disjoint subsets:

$$
\mathrm{I}=\bigcup_{k=1}^{K} \mathrm{I}^{k} \cup \mathrm{I}^{\mathrm{S}}, \quad \mathrm{O}=\bigcup_{k=1}^{K} \mathrm{O}^{k} \cup \mathrm{O}^{\mathrm{S}}
$$

The subsets $\mathrm{I}^{k}$ and $\mathrm{O}^{k}$ include the inputs and outputs that are specific (dedicated) to component processes $k \in \mathcal{K}=\{1, \ldots, K\}$. The subsets $\mathrm{I}^{\mathrm{S}}$ and $\mathrm{O}^{\mathrm{S}}$ include shared inputs and outputs, respectively. Each component process $k \in \mathcal{K}$ uses its component-specific inputs $\mathrm{I}^{k}$ and an unknown to us part of the shared inputs $\mathrm{I}^{\mathrm{S}}$ to produce the component-specific outputs $\mathrm{O}^{k}$ and an unknown part of the shared outputs $\mathrm{O}^{\mathrm{S}}$.

In order to simplify the discussion, we generically refer to the sets $\mathrm{I}^{\mathrm{S}}, \mathrm{O}^{\mathrm{S}}, \mathrm{I}^{k}$ and $\mathrm{O}^{k}, k \in \mathcal{K}$, as if they are non-empty sets. However, in practical applications, some of these sets may be empty. The results obtained in this paper are valid under the minimal assumption that each component process $k \in \mathcal{K}$ has at least one input and at least one output, and either can be component-specific or shared. Formally, the only assumption that we need for the validity of all our results is the following:

$$
\mathrm{I}^{k} \cup \mathrm{I}^{\mathrm{S}} \neq \varnothing, \quad \mathrm{O}^{k} \cup \mathrm{O}^{\mathrm{S}} \neq \varnothing, \quad \forall k \in \mathcal{K} .
$$

Taking into account (1), we state any $\operatorname{DMU}(X, Y)$ as follows:

$$
\left(\begin{array}{c}
X \\
Y
\end{array}\right)=\left(\begin{array}{c}
X^{1}, \ldots, X^{K}, X^{\mathrm{S}} \\
Y^{1}, \ldots, Y^{K}, Y^{\mathrm{S}}
\end{array}\right),
$$

where the superscripts relate the subvectors of inputs and outputs to the corresponding subsets defined by (1).

The described setting includes a wide range of practical scenarios. Let us consider some of them in the context of efficiency assessment of universities. This discussion is also aligned to the application in Section 9.

We take into account that each university consists of several academic departments. For homogeneity reasons, we classify all departments into three groups $k \in \mathcal{K}=\{1,2,3\}$ : medicine, science and non-science (e.g., social sciences and arts) departments. Each group has its own approaches to 
Podinovski: Technologies with Multiple Component Processes

teaching and research, requires different resources and is regarded as a component process within the university. Each group (process) uses academic staff, departmental expenditures (excluding academic staff) and a certain part of centralized expenditures (incurred at the university level) as inputs. Also, each group teaches undergraduate and postgraduate students and produces published research considered as outputs.

Depending on the availability of data, several scenarios arise. If all inputs and outputs are known exactly, including the allocation of centralized expenditure and published papers to the departments, we have only component-specific inputs and outputs, and no shared measures. In this case, the sets $\mathrm{I}^{k}$ and $\mathrm{O}^{k}$ include all inputs and outputs of each process $k \in \mathcal{K}$, and the sets of shared inputs and outputs $\mathrm{I}^{\mathrm{S}}$ and $\mathrm{O}^{\mathrm{S}}$ are empty.

Now assume that the centralized expenditures are reported in total and their allocation to the departments (or the three groups of departments) is unknown. In this case, the allocated parts of the centralized expenditure are removed from the component-specific sets ${ }^{k}$. Instead, their total is now considered as a shared input included in the set $\mathrm{I}^{\mathrm{S}}$. Similarly, if the number of published papers is known only in total for the whole university and not at the departmental level, this output is considered as shared and is included in the set $\mathrm{O}^{\mathrm{S}}$.

An interesting special case arises in which all inputs or all outputs, or even both, are reported in total. For example, suppose that we do not have information about the academic staff and other expenditures at the departmental level. Instead, we only have the total expenditure of the whole university (including staff costs) whose allocation to the departments is unknown. At the same time, we may still have component-specific information about the students. For example, a similar situation is considered in the application to universities reported by Thanassoulis et al. (2011). In the described case, the component-specific sets $\mathrm{I}^{k}$ are empty for all $k \in \mathcal{K}$, and the overall expenditure is the shared input included in the set $\mathrm{I}^{\mathrm{S}}$. (As a variant, we may have two shared inputs included in the set $\mathrm{I}^{\mathrm{S}}$, the first representing total academic staff and the second representing total other expenditure, both available only in aggregate, at the university level.) We consider all such special cases without component-specific inputs or outputs in Section 7. 
Note that, in all described scenarios, conditions (2) are satisfied, i.e., each component process has at least one input and at least one output. This is the only assumption that we require, and the models developed in this paper correctly represent the corresponding multi-component technology.

Further note that, in the described university setting, all three component processes (groups of department) have a similar input and output structure. In particular, all have undergraduate and postgraduate students and publications as outputs. However, in general, we make no assumption that the sets of component-specific inputs $\mathrm{I}^{k}$ and outputs $\mathrm{O}^{k}$ have a similar structure for all $k \in \mathcal{K}$. For example, in applications to agricultural farms, we may consider crop and livestock production as two component processes. These two processes, while possibly sharing costs and labor, may also have several mutually unrelated component-specific inputs and outputs, such as the use of fertilisers or milk production that would appear, as input and output, only in one of the processes.

REMARK 1. Let us comment on the possibility of incorporation of bad outputs (and, similarly, bad inputs) in the models developed in this paper. Many environmental applications include pollution as a bad output, and there is extensive literature on the modeling of such outputs (see, e.g., Dakpo et al. 2016 and Pham and Zelenyuk 2019). In a multi-component setting, the exact allocation of polluting levels to individual component processes may be problematic, and such bad outputs may be regarded as shared bad outputs. However, such component-specific or shared bad outputs do not satisfy Axiom 2 of free disposability (as outputs), and the models developed in this paper based on all stated axioms are not designed to incorporate them.

A similar situation arises in many applications of DEA with non-material bad outputs and inputs which are often treated as normal inputs and outputs, respectively (Dyson et al. 2001). The models developed in this paper allow the incorporation of component-specific and shared bad outputs and inputs treated in this way (by viewing them as inputs and outputs, respectively). The same approach can be used in environmental applications with polluting outputs if we assume that they satisfy Axiom 2 as inputs. However, such assumption for polluting outputs is not universally accepted, in particular, because of the material balance concerns (for a review, see Pham and Zelenyuk 2019). 
Podinovski: Technologies with Multiple Component Processes

Article submitted to Operations Research; manuscript no. (Please, provide the mansucript number!)

\section{Convexity of Technologies with Component Processes}

Below we explore the idea of defining convex combinations of DMUs in which their component processes are combined in convex combinations independently of the other component processes. The main difficulty here is the treatment of the shared inputs and outputs, as we do not know in which proportions such inputs and outputs are actually allocated to the component processes.

\subsection{A Simple Illustrative Case}

We first consider a simplified setting. Suppose that technology $T$ has only two component processes $k=1,2$. Consider the two DMUs $\left(\tilde{X}_{1}, \tilde{Y}_{1}\right)$ and $\left(\tilde{X}_{2}, \tilde{Y}_{2}\right)$ in $T$ stated as follows:

$$
\left(\begin{array}{c}
\tilde{X}_{l} \\
\tilde{Y}_{l}
\end{array}\right)=\left(\begin{array}{c}
\tilde{X}_{l}^{1}, \tilde{X}_{l}^{2}, \tilde{X}_{l}^{\mathrm{S}} \\
\tilde{Y}_{l}^{1}, \tilde{Y}_{l}^{2}, \tilde{Y}_{l}^{\mathrm{S}}
\end{array}\right), \quad l=1,2 .
$$

To relate to a practical setting, we may link this setup to the discussion of inputs and outputs in Section 2. For example, we may think of the DMUs $\left(\tilde{X}_{l}, \tilde{Y}_{l}\right)$ in $(4)$ as universities with two component processes: science $(k=1)$ and non-science $(k=2)$ departments. The inputs $\tilde{X}_{l}^{1}$ and $\tilde{X}_{l}^{2}$ are component-specific and represent academic staff at the science and non-science departments. Similarly, the outputs $\tilde{Y}_{l}^{1}$ and $\tilde{Y}_{l}^{2}$ are also component-specific and represent students at the science and non-science departments (we may count undergraduate and postgraduate students as separate components of the output vectors $\tilde{Y}_{l}^{1}$ and $\tilde{Y}_{l}^{2}$, or in total as their single components). The input $\tilde{X}_{l}^{\mathrm{S}}$ represents total expenditure of the university (excluding academic staff costs) and the output $\tilde{Y}_{l}^{S}$ represents total publications. We assume that the allocation of these two measures to science and non-science departments is unknown and treat both as shared measures.

First assume that there are no shared inputs and outputs. In this case, the vectors $\tilde{X}_{l}^{\mathrm{S}}$ and $\tilde{Y}_{l}^{\mathrm{S}}$ are removed from (4) and technology $T$ is decomposed into two independent separate components $T^{k}, k=1,2$, each using its own inputs $\mathrm{I}^{k}$ to produce outputs $\mathrm{O}^{k}$. This allows us to define convex combinations of the DMUs (4) using independent vectors of weighting coefficients $\lambda^{k}=\left(\lambda_{1}^{k}, \lambda_{2}^{k}\right) \geq 0$ (where $\lambda_{1}^{k}+\lambda_{2}^{k}=1$ ) for their component processes $k=1,2$. The resulting DMU is defined as follows:

$$
\left(\begin{array}{c}
\hat{X} \\
\hat{Y}
\end{array}\right)=\left(\begin{array}{c}
\lambda_{1}^{1} \tilde{X}_{1}^{1}+\lambda_{2}^{1} \tilde{X}_{2}^{1}, \lambda_{1}^{2} \tilde{X}_{1}^{2}+\lambda_{2}^{2} \tilde{X}_{2}^{2} \\
\lambda_{1}^{1} \tilde{Y}_{1}^{1}+\lambda_{2}^{1} \tilde{Y}_{2}^{1}, \lambda_{1}^{2} \tilde{Y}_{1}^{2}+\lambda_{2}^{2} \tilde{Y}_{2}^{2}
\end{array}\right) .
$$


Now consider the general case of (4) in which we have both shared inputs and outputs. To simplify discussion, we assume that there is a single shared input and a single shared output, i.e., each vector $\tilde{X}_{l}^{\mathrm{S}}$ and $\tilde{Y}_{l}^{\mathrm{S}}$ in (4) has a single component. (This assumption is not essential and is removed in the general case considered in the next section.) Note that we can no longer define the convex combinations of the DMUs $\left(\tilde{X}_{1}, \tilde{Y}_{1}\right)$ and $\left(\tilde{X}_{2}, \tilde{Y}_{2}\right)$ as in (5) because of the presence of the shared inputs and outputs. Let us show how this problem can be overcome.

Let $\alpha_{l}^{1}$ and $\alpha_{l}^{2}$ be the (unknown to us) proportions of the shared input $\tilde{X}_{l}^{\mathrm{S}}$ used by the DMUs $\left(\tilde{X}_{l}, \tilde{Y}_{l}\right), l=1,2$, in their component processes $k=1,2$, respectively. Similarly, let $\beta_{l}^{1}$ and $\beta_{l}^{2}$ be the (unknown to us) proportions of the shared output $\tilde{Y}_{l}^{\mathrm{S}}$ produced by the component processes $k=1,2$ of these DMUs, respectively. (We obviously require that $\alpha_{l}^{k}, \beta_{l}^{k} \geq 0$ for all $k$ and $l$, and $\alpha_{l}^{1}+\alpha_{l}^{2}=1$ and $\beta_{l}^{1}+\beta_{l}^{2}=1$, for $l=1,2$.) Therefore, for example, DMU $\left(\tilde{X}_{1}, \tilde{Y}_{1}\right)$ uses the amount $\alpha_{1}^{1} \tilde{X}_{1}^{\mathrm{S}}$ of its shared input in the first process and $\alpha_{1}^{2} \tilde{X}_{1}^{\mathrm{S}}$ in the second process, and $\alpha_{1}^{1} \tilde{X}_{1}^{\mathrm{S}}+\alpha_{1}^{2} \tilde{X}_{1}^{\mathrm{S}}=\tilde{X}_{1}^{\mathrm{S}}$.

With the shared input and output (hypothetically) allocated to the two processes, we can now construct the convex combinations of these processes of the two DMUs (4), taken with the weights $\lambda^{k}=\left(\lambda_{1}^{k}, \lambda_{2}^{k}\right) \geq 0, k=1,2$. We can subsequently aggregate the parts of the shared input and the shared output used by the two processes to the single shared input $\tilde{X}^{\mathrm{S}}$ and output $\tilde{Y}^{\mathrm{S}}$. The resulting DMU is stated as $(\hat{X}, \hat{Y})=\left(\hat{X}^{1}, \hat{X}^{2}, \hat{X}^{\mathrm{s}}, \hat{Y}^{1}, \hat{Y}^{2}, \hat{Y}^{\mathrm{S}}\right)$, where

$$
\begin{aligned}
& \hat{X}^{k}=\lambda_{1}^{k} \tilde{X}_{1}^{k}+\lambda_{2}^{k} \tilde{X}_{2}^{k}, \quad k=1,2, \\
& \hat{X}^{\mathrm{S}}=\lambda_{1}^{1} \alpha_{1}^{1} \tilde{X}_{1}^{\mathrm{S}}+\lambda_{1}^{2} \alpha_{1}^{2} \tilde{X}_{1}^{\mathrm{S}}+\lambda_{2}^{1} \alpha_{2}^{1} \tilde{X}_{2}^{\mathrm{S}}+\lambda_{2}^{2} \alpha_{2}^{2} \tilde{X}_{2}^{\mathrm{S}}, \\
& \hat{Y}^{k}=\lambda_{1}^{k} \tilde{Y}_{1}^{k}+\lambda_{2}^{k} \tilde{Y}_{2}^{k}, \quad k=1,2, \\
& \hat{Y}^{\mathrm{S}}=\lambda_{1}^{1} \beta_{1}^{1} \tilde{Y}_{1}^{\mathrm{S}}+\lambda_{1}^{2} \beta_{1}^{2} \tilde{Y}_{1}^{\mathrm{S}}+\lambda_{2}^{1} \beta_{2}^{1} \tilde{Y}_{2}^{\mathrm{S}}+\lambda_{2}^{2} \beta_{2}^{2} \tilde{Y}_{2}^{\mathrm{S}} .
\end{aligned}
$$

Note that equalities (6a) and (6c) are a restatement of (5). Assuming that the components $T^{1}$ and $T^{2}$ of technology $T$ are convex, the above DMU $(\hat{X}, \hat{Y})$ must be in technology $T$. Of course, because we do not know the actual proportions $\alpha_{l}^{k}$ and $\beta_{l}^{k}, k, l=1,2$, used by the two DMUs $\left(\tilde{X}_{1}, \tilde{Y}_{1}\right)$ and $\left(\tilde{X}_{2}, \tilde{Y}_{2}\right)$, their combination $(\hat{X}, \hat{Y})$ in $(6)$ cannot be calculated. 
Let us identify the largest shared input $\hat{X}^{\mathrm{S}}$ and the smallest shared output $\hat{Y}^{\mathrm{S}}$ that can be obtained from (6), by considering all possible allocation vectors $\left(\alpha_{l}^{1}, \alpha_{l}^{2}\right)$ and $\left(\beta_{l}^{1}, \beta_{l}^{2}\right), l=1,2$. This task is straightforward. Consider the sum $\left(\lambda_{1}^{1} \alpha_{1}^{1}+\lambda_{1}^{2} \alpha_{1}^{2}\right) \tilde{X}_{1}^{S}$ of the first two terms on the right-hand side of $(6 \mathrm{~b})$. The maximum of this sum with respect to $\left(\alpha_{1}^{1}, \alpha_{1}^{2}\right)$ is equal to $\max \left\{\lambda_{1}^{1}, \lambda_{1}^{2}\right\} \tilde{X}_{1}^{\mathrm{S}}$. It is attained at $\left(\alpha_{1}^{1}, \alpha_{1}^{2}\right)=(1,0)$ if $\lambda_{1}^{1} \geq \lambda_{1}^{2}$, and at $\left(\alpha_{1}^{1}, \alpha_{1}^{2}\right)=(0,1)$ otherwise. Similarly, the maximum of the sum $\left(\lambda_{2}^{1} \alpha_{2}^{1} \tilde{+} \lambda_{2}^{2} \alpha_{2}^{2} \tilde{)} X_{2}^{\mathrm{S}}\right.$ of the last two terms in (6b) is equal to $\max \left\{\lambda_{2}^{1}, \lambda_{2}^{2}\right\} \tilde{X}_{2}^{\mathrm{S}}$.

For the shared outputs, the minimum of the sum $\left(\lambda_{1}^{1} \beta_{1}^{1}+\lambda_{1}^{2} \beta_{1}^{2}\right) \tilde{Y}_{1}^{\mathrm{S}}$ of the first two terms on the right-hand side of $(6 \mathrm{~d})$ is equal to $\min \left\{\lambda_{1}^{1}, \lambda_{1}^{2}\right\} \tilde{Y}_{1}^{\mathrm{S}}$. If $\lambda_{1}^{1} \geq \lambda_{1}^{2}$, it is attained at $\left(\beta_{1}^{1}, \beta_{1}^{2}\right)=(0,1)$. Otherwise, it is attained at $\left(\beta_{1}^{1}, \beta_{1}^{2}\right)=(1,0)$. Similarly, the minimum of the sum $\left(\lambda_{2}^{1} \beta_{2}^{1}+\lambda_{2}^{2} \beta_{2}^{2}\right) \tilde{Y}_{2}^{\mathrm{S}}$ of the last two terms is equal to $\min \left\{\lambda_{2}^{1}, \lambda_{2}^{2}\right\} \tilde{Y}_{2}^{\mathrm{S}}$.

Using formula (6) with the described worst-case allocation vectors $\left(\alpha_{l}^{1}, \alpha_{l}^{2}\right)$ and $\left(\beta_{l}^{1}, \beta_{l}^{2}\right), l=1,2$, we obtain the DMU $(\tilde{X}, \tilde{Y})=\left(\tilde{X}^{1}, \tilde{X}^{2}, \tilde{X}^{\mathrm{S}}, \tilde{Y}^{1}, \tilde{Y}^{2}, \tilde{Y}^{\mathrm{S}}\right)$ whose components are defined as follows:

$$
\begin{aligned}
& \tilde{X}^{k}=\lambda_{1}^{k} \tilde{X}_{1}^{k}+\lambda_{2}^{k} \tilde{X}_{2}^{k}, \quad k=1,2, \\
& \tilde{X}^{\mathrm{S}}=\max \left\{\lambda_{1}^{1}, \lambda_{1}^{2}\right\} \tilde{X}_{1}^{\mathrm{S}}+\max \left\{\lambda_{2}^{1}, \lambda_{2}^{2}\right\} \tilde{X}_{2}^{\mathrm{S}}, \\
& \tilde{Y}^{k}=\lambda_{1}^{k} \tilde{Y}_{1}^{k}+\lambda_{2}^{k} \tilde{Y}_{2}^{k}, \quad k=1,2, \\
& \tilde{Y}^{\mathrm{S}}=\min \left\{\lambda_{1}^{1}, \lambda_{1}^{2}\right\} \tilde{Y}_{1}^{\mathrm{S}}+\min \left\{\lambda_{2}^{1}, \lambda_{2}^{2}\right\} \tilde{Y}_{2}^{\mathrm{S}} .
\end{aligned}
$$

Observe that $\operatorname{DMU}(\tilde{X}, \tilde{Y})$ is defined without any information about the actual allocation vectors $\left(\alpha_{l}^{1}, \alpha_{l}^{2}\right)$ and $\left(\beta_{l}^{1}, \beta_{l}^{2}\right), l=1,2$. By construction, it has the largest shared input and the smallest shared output among all DMUs $(\hat{X}, \hat{Y})$ that can in principle be obtained by formula $(6)$, for all such possible allocation vectors. Therefore, the DMU $(\hat{X}, \hat{Y})$ defined by $(6)$ weakly dominates DMU $(\tilde{X}, \tilde{Y})$ defined by $(7)$. Indeed, we have $\hat{X}^{k}=\tilde{X}^{k}$ and $\hat{Y}^{k}=\tilde{Y}^{k}$, for $k=1,2$, and $\hat{X}^{\mathrm{S}} \leq \tilde{X}^{\mathrm{S}}$ and $\hat{Y}^{\mathrm{S}} \geq \tilde{Y}^{\mathrm{S}}$. Assuming that technology $T$ satisfies Axiom 2 of free disposability (it suffices that only the shared inputs and outputs are freely disposable), we conclude that $(\tilde{X}, \tilde{Y}) \in T$.

Note that, if in (7) we use the same vectors $\lambda^{1}=\lambda^{2}$, the resulting $\operatorname{DMU}(\tilde{X}, \tilde{Y})$ becomes a conventional convex combination of DMUs $\left(\tilde{X}_{1}, \tilde{Y}_{1}\right)$ and $\left(\tilde{X}_{2}, \tilde{Y}_{2}\right)$. However, the use of different vectors $\lambda^{1}$ and $\lambda^{2}$ for the two component processes allows us to generate DMUs $(\tilde{X}, \tilde{Y})$ that cannot 
Table 1 DMUs in Example 1

\begin{tabular}{lcccccc}
\hline DMU & $X^{1}$ & $X^{2}$ & $X^{\mathrm{S}}$ & $Y^{1}$ & $Y^{2}$ & $Y^{\mathrm{S}}$ \\
\hline$A$ & 1 & 2 & 1 & 2 & 1 & 1 \\
$B$ & 2 & 1 & 1 & 1 & 2 & 1 \\
$C: \lambda^{1}=(0.5,0.5), \lambda^{2}=(0,1)$ & 1.5 & 1 & 1.5 & 1.5 & 2 & 0.5 \\
$D: \lambda^{1}=(0.6,0.4), \lambda^{2}=(0.2,0.8)$ & 1.4 & 1.2 & 1.4 & 1.6 & 1.8 & 0.6 \\
$E: \lambda^{1}=(1,0), \lambda^{2}=(0,1)$ & 1 & 1 & 2 & 2 & 2 & 0 \\
\hline
\end{tabular}

be obtained as the conventional convex combination of DMUs $\left(\tilde{X}_{1}, \tilde{Y}_{1}\right)$ and $\left(\tilde{X}_{2}, \tilde{Y}_{2}\right)$. We illustrate this observation by the following example.

Example 1. Table 1 shows DMUs $A$ and $B$ in a technology with two component processes $k=1,2$. The inputs $X^{1}$ and $X^{2}$ and outputs $Y^{1}$ and $Y^{2}$ are component-specific. The input $X^{\mathrm{S}}$ and output $Y^{\mathrm{S}}$ are shared. DMUs $C, D$ and $E$ are obtained by formula (7) for different vectors $\lambda^{1}=\left(\lambda_{A}^{1}, \lambda_{B}^{1}\right)$ and $\lambda^{2}=\left(\lambda_{A}^{2}, \lambda_{B}^{2}\right)$ used to combine component processes 1 and 2 of DMUs $A$ and $B$.

Consider, for example, DMU $E$. Because $\lambda_{A}^{1}=1$ and $\lambda_{B}^{1}=0$, the component process 1 of DMU $E$ is defined by DMU $A$. Similarly, because $\lambda_{A}^{2}=0$ and $\lambda_{B}^{2}=1$, the component process 2 of DMU $E$ is defined by DMU $B$. Consequently, the input $X^{1}$ and output $Y^{1}$ of DMUs $E$ and $A$ are identical. Similarly, the input $X^{2}$ and output $Y^{2}$ of DMUs $E$ and $B$ are the same.

Consider the shared input $X^{\mathrm{S}}$. In the worst case, DMU $A$ may use its shared input $X^{\mathrm{S}}=1$ entirely for process 1 (which would correspond to the allocation $\alpha_{A}^{1}=1$ and $\alpha_{A}^{2}=0$ ). Similarly, DMU $B$ may use its entire shared input $X^{\mathrm{S}}=1$ for process 2 only (which would correspond to $\alpha_{B}^{1}=0$ and $\left.\alpha_{B}^{2}=1\right)$. Therefore, in order for DMU $E$ to have sufficient shared input for both processes (the first of which is taken from DMU $A$ and the second from DMU $B$ ) even in the worst case, it needs the sum of the shared inputs $X^{\mathrm{S}}$ of the DMUs $A$ and $B$, which is equal to 2 .

Consider the shared output $Y^{\mathrm{S}}$. It is possible that DMU $A$ does not produce this output in its process 1 and DMU $B$ does not produce it in its process 2. Accounting for this possibility as the worst case, formula (7) defines the shared output of the combined DMU $E$ as zero.

It is straightforward to verify that DMUs $C, D$ and $E$ are not in the standard VRS technology 
Podinovski: Technologies with Multiple Component Processes

Article submitted to Operations Research; manuscript no. (Please, provide the mansucript number!)

generated by DMUs $A$ and $B$. In other words, DMUs $C, D$ and $E$ cannot be obtained as a convex combination of $A$ and $B$ and are not outperformed (dominated) by any such combination.

\subsection{The General Setting}

We now extend the treatment of convex combinations of component processes developed in the previous section, to the general setting. Namely, we allow any finite number $K$ of component processes and any finite number of shared inputs and outputs in the sets $\mathrm{I}^{\mathrm{S}}$ and $\mathrm{O}^{\mathrm{S}}$.

Consider a finite number $L \geq 2$ of DMUs in technology $T$ stated as follows:

$$
\left(\begin{array}{c}
\tilde{X}_{l} \\
\tilde{Y}_{l}
\end{array}\right)=\left(\begin{array}{c}
\tilde{X}_{l}^{1}, \ldots, \tilde{X}_{l}^{K}, \tilde{X}_{l}^{\mathrm{S}} \\
\tilde{Y}_{l}^{1}, \ldots, \tilde{Y}_{l}^{K}, \tilde{Y}_{l}^{\mathrm{S}}
\end{array}\right), \quad l=1, \ldots, L .
$$

Consider any $\operatorname{DMU}\left(\tilde{X}_{l}, \tilde{Y}_{l}\right), l=1, \ldots, L$, and any of its shared inputs $\tilde{X}_{l i}^{\mathrm{S}}$, where $i \in \mathrm{I}^{\mathrm{S}}$. We assume that this input is actually allocated to the component processes $k=1, \ldots, K$ of DMU $\left(\tilde{X}_{l}, \tilde{Y}_{l}\right)$ in the (unknown to us) proportions described by the vector $\alpha_{l i}=\left(\alpha_{l i}^{1}, \ldots, \alpha_{l i}^{K}\right) \in \mathbb{R}_{+}^{K}$, such that $1^{\top} \alpha_{l i}=1$. Similarly, we assume that any shared output $\tilde{Y}_{l r}^{\mathrm{S}}$ of $\mathrm{DMU}\left(\tilde{X}_{l}, \tilde{Y}_{l}\right)$, where $r \in \mathrm{O}^{\mathrm{S}}$, is allocated to its $K$ processes in the unknown proportions described by the vector $\beta_{l r}=\left(\beta_{l r}^{1}, \ldots, \beta_{l r}^{K}\right) \in \mathbb{R}_{+}^{K}$, such that $1^{\top} \beta_{l r}=1$. The vectors $\alpha_{l i}$ and $\beta_{l r}$ are assumed to be generally different for different DMUs $l=1, \ldots, L$, shared inputs $i \in \mathrm{I}^{\mathrm{S}}$ and shared outputs $r \in \mathrm{O}^{\mathrm{S}}$, respectively.

We can now consider component subtechnologies $T^{k}, k=1, \ldots, K$. In each $T^{k}$ and for each $l=$ $1, \ldots, L$, the input vector $\tilde{X}_{l}^{k}$ and the allocated parts of the shared inputs $\alpha_{l i}^{k} \tilde{X}_{l i}^{\mathrm{S}}, i \in \mathrm{I}^{\mathrm{S}}$, are used to produce the output vector $\tilde{Y}_{l}^{k}$ and the allocated parts of the shared outputs $\beta_{l r}^{k} \tilde{Y}_{l r}^{\mathrm{S}}, r \in \mathrm{O}^{\mathrm{S}}$.

Consider constructing convex combinations of all DMUs $\left(\tilde{X}_{l}, \tilde{Y}_{l}\right), l=1, \ldots, L$, defined by $(8)$, using component-specific vectors $\lambda^{k}=\left(\lambda_{1}^{k}, \ldots, \lambda_{L}^{k}\right) \geq 0$, such that $1^{\top} \lambda^{k}=1, k=1, \ldots, K$. Subsequently aggregating the shared inputs and outputs, we obtain the following DMU:

$$
\begin{aligned}
& \hat{X}^{k}=\sum_{l=1}^{L} \lambda_{l}^{k} \tilde{X}_{l}^{k}, \quad \forall k, \\
& \hat{X}_{i}^{\mathrm{S}}=\sum_{k=1}^{K} \sum_{l=1}^{L} \lambda_{l}^{k} \alpha_{l i}^{k} \tilde{X}_{l i}^{\mathrm{S}}, \quad \forall i \in \mathrm{I}^{\mathrm{S}},
\end{aligned}
$$




$$
\begin{aligned}
& \hat{Y}^{k}=\sum_{l=1}^{L} \lambda_{l}^{k} \tilde{Y}_{l}^{k}, \quad \forall k, \\
& \hat{Y}_{r}^{\mathrm{S}}=\sum_{k=1}^{K} \sum_{l=1}^{L} \lambda_{l}^{k} \beta_{l r}^{k} \tilde{Y}_{l r}^{\mathrm{S}}, \quad \forall r \in \mathrm{O}^{\mathrm{S}} .
\end{aligned}
$$

The interior sums in $(9 \mathrm{~b})$ and $(9 \mathrm{~d})$ (taken with respect to $l$ ) represent convex combinations of the shared inputs and outputs allocated to each process $k=1, \ldots, K$. The exterior sums (taken with respect to $k$ ) represent the total amounts of such inputs and outputs aggregated across all component processes.

Because we do not know the proportions $\alpha_{l i}^{k}$ and $\beta_{l r}^{k}$, used by the DMUs (8), their convex combination $(\hat{X}, \hat{Y})$ cannot actually be calculated. However, using formula (9) we can evaluate the largest amount $\hat{X}_{i}^{\mathrm{S}}$ of each shared input $i \in \mathrm{I}^{\mathrm{S}}$, and the smallest amount $\hat{Y}_{r}^{\mathrm{S}}$ of each shared output $r \in \mathrm{O}^{\mathrm{S}}$, over the set of all possible allocations $\alpha_{l i}^{k}$ and $\beta_{l r}^{k}$, that the $\operatorname{DMU}(\hat{X}, \hat{Y})$ can have.

Proposition 1. For each $i \in \mathrm{I}^{\mathrm{S}}$, the maximum of $\hat{X}_{i}^{\mathrm{S}}$ over the set of all possible allocations $\alpha_{l i}^{k}$ of the shared inputs is attained and is equal to $\tilde{X}_{i}^{\mathrm{S}}=\sum_{l=1}^{L} \max _{k \in \mathcal{K}}\left\{\lambda_{l}^{k}\right\} X_{l i}^{\mathrm{S}}$. Similarly, for any $r \in \mathrm{O}^{\mathrm{S}}$, the minimum of $\hat{Y}_{r}^{S}$ over the set of all possible allocations $\beta_{l r}^{k}$ of the shared outputs is attained and is equal to $\tilde{Y}_{r}^{\mathrm{S}}=\sum_{l=1}^{L} \min _{k \in \mathcal{K}}\left\{\lambda_{l}^{k}\right\} Y_{l r}^{\mathrm{S}}$.

The proofs of this and the other statements are given in Appendix A.

In line with Proposition 1, define $\operatorname{DMU}(\tilde{X}, \tilde{Y})$ as follows:

$$
\begin{aligned}
& \tilde{X}^{k}=\sum_{l=1}^{L} \lambda_{l}^{k} \tilde{X}_{l}^{k}, \quad \forall k, \\
& \tilde{X}^{\mathrm{S}}=\sum_{l=1}^{L} \max _{k \in \mathcal{K}}\left\{\lambda_{l}^{k}\right\} \tilde{X}_{l}^{\mathrm{S}}, \\
& \tilde{Y}^{k}=\sum_{l=1}^{L} \lambda_{l}^{k} \tilde{Y}_{l}^{k}, \quad \forall k, \\
& \tilde{Y}^{\mathrm{S}}=\sum_{l=1}^{L} \min _{k \in \mathcal{K}}\left\{\lambda_{l}^{k}\right\} \tilde{Y}_{l}^{\mathrm{S}} .
\end{aligned}
$$

By Proposition 1, DMU $(\tilde{X}, \tilde{Y})$ defined by (10) has the maximal levels of the shared inputs and the minimal levels of the shared outputs among all DMUs $(\hat{X}, \hat{Y})$ obtained by formula $(9)$. Therefore, DMU $(\tilde{X}, \tilde{Y})$ is the most conservative DMU that can be obtained in this way. 
Podinovski: Technologies with Multiple Component Processes

We do not know the exact $\operatorname{DMU}(\hat{X}, \hat{Y}) \in T$ defined by (9) for which the exact allocations $\alpha_{l i}^{k}$ and $\beta_{l r}^{k}$ of the shared inputs and outputs are required. However, by Proposition 1, this unknown $\operatorname{DMU}(\hat{X}, \hat{Y})$ dominates (outperforms) the $\operatorname{DMU}(\tilde{X}, \tilde{Y})$ defined by (10). Assuming that technology $T$ is freely disposable with respect to the shared inputs and outputs, we conclude that $(\tilde{X}, \tilde{Y}) \in T$.

It is worth highlighting the fact that the combined DMUs $(\tilde{X}, \tilde{Y})$ obtained by formula (10) include all standard convex combinations of DMUs (8). Indeed, the latter correspond to the special case in which we use the same vector $\lambda^{k}$ for all component processes $k=1, \ldots, K$.

\subsection{A Formal Axiomatic Statement}

As argued in the previous section, for a convex technology $T$ with $K$ component processes, we should be able to define convex combinations of its DMUs with the vectors of weights $\lambda^{k}$ that are generally different for different component processes. A particular difficulty in this case arises from the fact that we do not know the exact proportions in which the shared inputs and outputs are allocated to the component processes at each DMU. This difficulty is overcome by defining the convex combination using the most conservative $\operatorname{DMU}(\tilde{X}, \tilde{Y})$ as in $(10)$ which does not depend on the actual allocations of the shared measures.

The described worst-case approach to the definition of the $\operatorname{DMU}(\tilde{X}, \tilde{Y})$ is justified assuming that technology $T$ is freely disposable in the sense of Axiom 2. Therefore, the axiom of convexity stated below should be assumed only in conjunction with Axiom 2 (or its weaker analogue requiring that $T$ be freely disposable at least with respect to all of its shared inputs and outputs).

Axiom $3^{*}$ (Multi-Component Convexity). Let DMUs $\left(\tilde{X}_{l}, \tilde{Y}_{l}\right), l=1, \ldots, L$, defined by $(8)$ be in technology $T$. Consider any $K$ vectors $\lambda^{k} \in \mathbb{R}_{+}^{L}$ such that $1^{\top} \lambda^{k}=1$, for all $k=1, \ldots, K$. Then DMU $(\tilde{X}, \tilde{Y})$ defined by $(10)$ is in $T$.

As noted, if the vectors of weights $\lambda^{k}$ are the same for all component processes $k$, the DMU $(\tilde{X}, \tilde{Y})$ in $(10)$ is a standard convex combination of DMUs (8). Therefore, if technology $T$ satisfies Axiom $3^{*}$, then it also satisfies Axiom 3, and the latter does not need to be assumed if the former is assumed. 
REMARK 2. It is worth highlighting the conceptual similarity between the worst-case approach on which Axiom $3^{*}$ is based and the minimum extrapolation principle used in the axiomatic definition of technologies. Indeed, both represent the underlying principle that the model of technology should include only those DMUs which are required to satisfy the assumed information about the production process (e.g., stated as axioms) and should not include any other arbitrary DMUs. In the case of multi-component convexity, we do not know the exact proportions $\alpha_{l i}^{k}$ and $\beta_{l r}^{k}$ in which the shared inputs $i$ and outputs $r$ are actually allocated to each process $k$ of each DMU $\left(\tilde{X}_{l}, \tilde{Y}_{l}\right)$, and therefore we do not know their true multi-component convex combination $(\hat{X}, \hat{Y})$ defined by (9). However, as follows from Proposition 1, regardless of the unknown to us true convex combination $(\hat{X}, \hat{Y})$, the DMU $(\tilde{X}, \tilde{Y})$ defined by (10) must be included in the freely disposable technology with any such unknown $(\hat{X}, \hat{Y})$, which is postulated by Axiom $3^{*}$. The same principle is employed in Axiom $4^{*}$ of multi-component scaling considered in Section 5.

The use of the worst-case and minimum extrapolation principles results in the smallest possible technology that is based on the available information about the production process and leads to the most conservative (highest possible) efficiency scores of the DMUs under the stated assumptions. This is consistent with the known principle of DEA according to which the efficiency is evaluated in the most favourable way for the DMU under the assessment. In Section 6, we observe how the principle of most favourable treatment manifests itself in the dual models based on multi-component technologies.

REMARK 3. Our basic assumption is that each DMU allocates all its shared inputs and outputs to different component processes, although in some unknown proportions. In some applications, we may also have inputs and outputs that cannot be allocated to component processes in principle.

An example of this is the joint inputs considered by Cherchye et al. (2013). Such inputs have a positive impact on all component processes but their allocation to the latter in different proportions is meaningless. For example, Podinovski et al. (2018) considers an application to secondary schools each consisting of three component processes representing different programs of study. A possible 
joint input in this case may be some positive socio-economic characteristic (e.g., a measure of affluence) of the catchment area that equally affects all programs. Similarly, an undesirable socioeconomic characteristic (e.g., a measure of deprivation) may be considered as a joint bad input and treated by the model as an output that cannot be allocated to component processes.

In other applications, inputs and outputs that cannot be divided between component processes may represent various environmental measures, availability of infrastructure, advantages of the location of a DMU, age characteristics of the population, and so on.

All production axioms assumed in this paper, including Axiom $3^{*}$ of multi-component convexity and a further Axiom 4* of scalability (stated in Section 5) remain valid if some or all of the shared inputs and outputs are jointly used and cannot be allocated in principle. Therefore, both the VRS and CRS models with component processes developed in this paper remain substantiated and valid in this case as well.

It should however be noted that, in applications with only joint inputs (that cannot be allocated in principle) and component-specific inputs and outputs, more suitable models would be those developed by Cherchye et al. (2013) specifically for this scenario. As follows from the example in Podinovski et al. (2018, p. 293), the assumption of joint inputs in the latter models results in a larger technology than the multi-component VRS and CRS technologies developed below, based on the worst-case assumption about the unknown allocation of the shared inputs and outputs.

\section{The VRS Technology with Multiple Component Processes}

Following the minimum extrapolation principle used by Banker et al. (1984), we define the multicomponent variable returns-to-scale (MVRS) technology $T_{\mathrm{MVRS}}$ as the smallest technology that satisfies Axioms 1, 2 and $3^{*}$. This leads to the following definition.

Definition 1. Technology $T_{\mathrm{MVRS}}$ is the intersection of all technologies $T \subset \mathbb{R}_{+}^{m+s}$ that satisfy Axioms 1, 2 and $3^{*}$.

It is straightforward to verify that technology $T_{\text {MVRS }}$ itself satisfies Axioms 1,2 and $3^{*}$ and is, therefore, the smallest among all technologies that satisfy the stated axioms. 
The following result gives an explicit statement of technology $T_{\mathrm{MVRS}}$.

TheOrem 1. Technology $T_{\text {MVRs }}$ is the set of all DMUs $(X, Y) \in \mathbb{R}_{+}^{m+s}$ for which there exist vectors $\lambda^{k} \in \mathbb{R}^{n}, k=1, \ldots, K$, such that the following conditions are true:

$$
\begin{aligned}
& \sum_{j=1}^{n} \lambda_{j}^{k} X_{j}^{k} \leq X^{k}, \quad \forall k, \\
& \sum_{j=1}^{n} \max _{k \in \mathcal{K}}\left\{\lambda_{j}^{k}\right\} X_{j}^{\mathrm{S}} \leq X^{\mathrm{S}}, \\
& \sum_{j=1}^{n} \lambda_{j}^{k} Y_{j}^{k} \geq Y^{k}, \quad \forall k \\
& \sum_{j=1}^{n} \min _{k \in \mathcal{K}}\left\{\lambda_{j}^{k}\right\} Y_{j}^{\mathrm{S}} \geq Y^{\mathrm{S}}, \\
& 1^{\top} \lambda^{k}=1, \quad \forall k \\
& \lambda^{k} \geq 0, \forall k .
\end{aligned}
$$

We now restate conditions (11) in a linearized form.

Proposition 2. Technology $T_{\text {MVRS }}$ is equivalently stated as the set of all DMUs $(X, Y) \in \mathbb{R}_{+}^{m+s}$ for which there exist vectors $\lambda^{k} \in \mathbb{R}^{n}, k=1, \ldots, K$, and $\lambda^{\max }, \lambda^{\min } \in \mathbb{R}^{n}$ such that

$$
\begin{aligned}
& \sum_{j=1}^{n} \lambda_{j}^{k} X_{j}^{k} \leq X^{k}, \quad \forall k, \\
& \sum_{j=1}^{n} \lambda_{j}^{\max } X_{j}^{\mathrm{S}} \leq X^{\mathrm{S}}, \\
& \sum_{j=1}^{n} \lambda_{j}^{k} Y_{j}^{k} \geq Y^{k}, \quad \forall k, \\
& \sum_{j=1}^{n} \lambda_{j}^{\min } Y_{j}^{\mathrm{S}} \geq Y^{\mathrm{S}}, \quad \forall j, k, \\
& \lambda_{j}^{\max } \geq \lambda_{j}^{k}, \quad \forall j, k, \\
& \lambda_{j}^{\min } \leq \lambda_{j}^{k}, \quad \forall k, \\
& 1^{\top} \lambda^{k}=1, \quad \forall k . \\
& \lambda^{\max }, \lambda^{\min }, \lambda^{k} \geq 0,
\end{aligned}
$$

Proposition 3. Technology $T_{\mathrm{MVRS}}$ is a polyhedral (and, therefore, convex and closed) set. 
The following result shows that the multi-component VRS technology $T_{\text {MVRS }}$ includes the standard VRS technology $T_{\mathrm{VRS}}$ of Banker et al. (1984) as a subset.

Proposition 4. $T_{\mathrm{VRS}} \subseteq T_{\mathrm{MVRS}}$.

Example 1 provides a simple illustration of Proposition 4. In this example, DMUs $C, D$ and $E$ are obtained from the observed DMUs $A$ and $B$ by Axiom $3^{*}$ and are therefore in the technology $T_{\text {MVRS }}$ generated by $A$ and $B$. However, as noted, these DMUs are not in technology $T_{\mathrm{VRS}}$. Proposition 4 is further illustrated by the application in Section 9 .

\section{The CRS Technology with Multiple Component Processes}

We now consider the case of a CRS technology in which several component processes are not only convex (as in the MVRS technology) but are also scalable independently of each other, i.e., we consider each component process as a CRS subtechnology. As in the case of MVRS, we again need to overcome the fact that we generally do not know the proportions of the shared inputs and outputs attributable to each of the component processes.

\subsection{Scalability of Component Processes}

The following idea was first employed by Podinovski et al. (2018) for the development of the multiple hybrid returns-to-scale technology. Consider a $\operatorname{DMU}(X, Y)$ stated as in (3) and suppose that we wish to scale its component processes $k$ with some generally different nonnegative scaling factors $\rho^{k}, k=1, \ldots, K$. Denote $\rho \in \mathbb{R}_{+}^{K}$ the vector of such scaling factors.

Suppose first that we do not have shared inputs and outputs. Then we can apply the scaling factors $\rho^{k}$ to the corresponding component-specific input and output vectors $X^{k}$ and $Y^{k}$, for all $k=1, \ldots, K$. As a result, $\mathrm{DMU}(X, Y)$ is transformed to

$$
\left(\begin{array}{c}
X(\rho) \\
Y(\rho)
\end{array}\right)=\left(\begin{array}{c}
\rho^{1} X^{1}, \ldots, \rho^{K} X^{K} \\
\rho^{1} Y^{1}, \ldots, \rho^{K} Y^{K}
\end{array}\right) .
$$

Now consider the general case which involves shared inputs and outputs. Consider the inputs first. Because we do not know the exact parts of the shared inputs allocated to each component 
process of DMU $(X, Y)$, and because these parts need to be scaled by generally different multipliers $\rho^{k}$, we assume the most conservative, or worst-case, scenario and scale all shared inputs by the maximum of all multipliers $\rho^{k}$. This means that, irrespective of the actual unknown allocation of the shared inputs to different component processes each of which is scaled with a different multiplier $\rho^{k}$, the resulting scaled processes have sufficient amounts of the shared inputs for their operations.

The case of shared outputs is similar. Because we do not know the proportions of the shared outputs attributable to each component process, we scale all shared outputs by the minimum of all multipliers $\rho^{k}$. This means that, irrespective of the actual contribution of each process to the shared outputs and irrespective of the fact that these processes are scaled by different multipliers, the resulting shared outputs can be produced.

To state the above idea formally as an axiom, define

$$
\rho^{\min }=\min \left\{\rho^{k} \mid k \in \mathcal{K}\right\}, \quad \rho^{\max }=\max \left\{\rho^{k} \mid k \in \mathcal{K}\right\} .
$$

In line with the above discussion, consider the DMU

$$
\left(\begin{array}{c}
X(\rho) \\
Y(\rho)
\end{array}\right)=\left(\begin{array}{c}
\rho^{1} X^{1}, \ldots, \rho^{K} X^{K}, \rho^{\max } X^{\mathrm{S}} \\
\rho^{1} Y^{1}, \ldots, \rho^{K} Y^{K}, \rho^{\min } Y^{\mathrm{S}}
\end{array}\right) .
$$

Axiom $4^{*}$ (Multi-Component Scalability). Let $(X, Y) \in T$. Then $(X(\rho), Y(\rho)) \in T$, for all $\rho \in \mathbb{R}_{+}^{K}$.

It is clear that the standard property of scalability stated by Axiom 4 is a special case of Axiom $4^{*}$ in which all components of the scaling vector $\rho$ are taken equal.

\subsection{The MCRS Technology}

We are now ready to define the CRS technology with multiple component processes. Each of these processes is considered convex and individually scalable. Therefore, the conventional axioms of convexity and scalability of Banker et al. (1984) are replaced by their multiple-component analogues, i.e., by Axioms $3^{*}$ and $4^{*}$.

We first observe that, although Axiom $3^{*}$ is generally stronger than Axiom 3 (in the sense that

the former implies the latter), according to the next result, the two axioms become equivalent if Axiom $4^{*}$ is assumed true. 
Proposition 5. Let technology $T$ satisfy Axiom $4^{*}$. Then $T$ satisfies Axiom 3 if and only if $T$ satisfies Axiom $3^{*}$.

Using the minimum extrapolation principle, define the multi-component constant returns-toscale (MCRS) technology $T_{\mathrm{MCRS}}$ as the smallest technology that satisfies Axioms $1,2,3^{*}$ and $4^{*}$.

Definition 2. Technology $T_{\mathrm{MCRS}}$ is the intersection of all technologies $T \subset \mathbb{R}_{+}^{m+s}$ that satisfy Axioms 1, 2, 3* and 4*. (Alternatively, by Proposition 5, we can require Axiom 3 instead of Axiom $3^{*}$.)

It is easy to prove that technology $T_{\mathrm{MCRS}}$ itself satisfies Axioms $1,2,3^{*}$ and $4^{*}$ and is, therefore, the smallest among all technologies that satisfy these axioms.

THEOREM 2. Technology $T_{\mathrm{MCRS}}$ is the set of all DMUs $(X, Y) \in \mathbb{R}_{+}^{m+s}$ for which there exist vectors $\lambda^{k} \in \mathbb{R}^{n}, k=1, \ldots, K$, such that the following conditions are true:

$$
\begin{aligned}
& \sum_{j=1}^{n} \lambda_{j}^{k} X_{j}^{k} \leq X^{k}, \quad \forall k, \\
& \sum_{j=1}^{n} \max _{k \in \mathcal{K}}\left\{\lambda_{j}^{k}\right\} X_{j}^{\mathrm{S}} \leq X^{\mathrm{S}}, \\
& \sum_{j=1}^{n} \lambda_{j}^{k} Y_{j}^{k} \geq Y^{k}, \quad \forall k, \\
& \sum_{j=1}^{n} \min _{k \in \mathcal{K}}\left\{\lambda_{j}^{k}\right\} Y_{j}^{\mathrm{S}} \geq Y^{\mathrm{S}}, \\
& \lambda^{k} \geq 0, \forall k .
\end{aligned}
$$

The following result restates conditions (14) in a linearized form.

Proposition 6. Technology $T_{\mathrm{MCRS}}$ is equivalently stated as the set of all DMUs $(X, Y) \in \mathbb{R}_{+}^{m+s}$ for which there exist vectors $\lambda^{k} \in \mathbb{R}^{n}, k=1, \ldots, K$, and $\lambda^{\max }, \lambda^{\min } \in \mathbb{R}^{n}$ such that 


$$
\begin{aligned}
& \sum_{j=1}^{n} \lambda_{j}^{k} X_{j}^{k} \leq X^{k}, \quad \forall k, \\
& \sum_{j=1}^{n} \lambda_{j}^{\max } X_{j}^{\mathrm{S}} \leq X^{\mathrm{S}}, \\
& \sum_{j=1}^{n} \lambda_{j}^{k} Y_{j}^{k} \geq Y^{k}, \quad \forall k, \\
& \sum_{j=1}^{n} \lambda_{j}^{\min } Y_{j}^{\mathrm{S}} \geq Y^{\mathrm{S}}, \\
& \lambda_{j}^{\max } \geq \lambda_{j}^{k}, \\
& \lambda_{j}^{\min } \leq \lambda_{j}^{k}, \\
& \lambda^{\max }, \lambda^{\min }, \lambda^{k} \geq 0, \quad \forall k .
\end{aligned}
$$

According to Theorem 2 and Proposition 6, the statements (14) and (15) of technology $T_{\text {MCRS }}$ are obtained from the corresponding statements (11) and (12) of technology $T_{\text {MvRs }}$, by removing all normalizing equalities (11e) and (12g) from the latter.

Proposition 7. Technology $T_{\mathrm{MCRS}}$ is a polyhedral (and, therefore, convex and closed) set.

Because Axioms $3^{*}$ and $4^{*}$ imply Axioms 3 and 4 , we have the following embedding involving the standard CRS technology $T_{\mathrm{CRS}}$ of Charnes et al. (1978).

Proposition 8. $T_{\mathrm{CRS}} \subseteq T_{\mathrm{MCRS}}$.

The established embedding is illustrated by the application in Section 9 .

\section{Envelopment and Multiplier Models}

In this section we consider the envelopment and multiplier models based on the MVRS and MCRS technologies. To be specific, we discuss the output radial measure of efficiency. Other measures, including the input radial measure of efficiency and the measures based on directional distance functions (Chambers et al. 1998), are unproblematic and can be considered in a similar way.

\subsection{The MVRS Envelopment Model}

Consider any $\mathrm{DMU}_{o}$ in the MVRS technology. Using the linearized statement (12) of this technology, we evaluate the output radial efficiency of $\mathrm{DMU}_{o}$ as the inverse of the optimal value $\eta^{*}$ of the linear program: 


$$
\begin{aligned}
\eta^{*}=\max & \eta \\
\text { s.t. } & \sum_{j=1}^{n} \lambda_{j}^{k} X_{j}^{k} \leq X_{o}^{k}, \quad \forall k, \\
& \sum_{j=1}^{n} \lambda_{j}^{\max } X_{j}^{\mathrm{S}} \leq X_{o}^{\mathrm{S}}, \\
& -\sum_{j=1}^{n} \lambda_{j}^{k} Y_{j}^{k}+\eta Y_{o}^{k} \leq 0, \quad \forall k, \quad \forall j, k, \\
& -\sum_{j=1}^{n} \lambda_{j}^{\min } Y_{j}^{\mathrm{S}}+\eta Y_{o}^{\mathrm{S}} \leq 0, \quad \forall j, k, \\
& \lambda_{j}^{k}-\lambda_{j}^{\max } \leq 0, \quad \forall k, \quad \\
& -\lambda_{j}^{k}+\lambda_{j}^{\min } \leq 0, \quad \forall k .
\end{aligned}
$$

In contrast with the standard output-oriented VRS model of Banker et al. (1984), the MVRS model (16) uses a separate variable vector $\lambda^{k}=\left(\lambda_{1}^{k}, \ldots, \lambda_{n}^{k}\right)$ with each component process $k$, and the normalizing equalities (16h) on the vectors $\lambda^{k}$ are stated separately for each process. This enables an independent construction of convex combinations for each component process and generally leads to a larger production technology compared to the standard VRS model.

The MVRS model (16) does not utilize any apportioning mechanism by which the shared inputs and outputs are allocated to different component processes. Inequalities (16f) imply that, for each $j \in J$, the variable $\lambda_{j}^{\max }$ is greater than or equal to the largest of the variables $\lambda_{j}^{k}$ taken across all component processes $k$. Similarly, inequalities $(16 \mathrm{~g})$ imply that the variable $\lambda_{j}^{\mathrm{min}}$ is less than or equal to the smallest of the variables $\lambda_{j}^{k}$ over all $k$. These variables $\lambda_{j}^{\max }$ and $\lambda_{j}^{\min }$ are used as the weights in the combinations of the shared inputs and outputs in constraints (16c) and (16e). As shown in the discussion of Axiom $3^{*}$, this guarantees that the composite DMU constructed on the left-hand side of model (16) is producible irrespective of the actual (but unknown to us) allocation of the shared inputs and outputs to different component processes at each observed DMU.

REMARK 4. Similar to the VRS model, the output radial projection $\left(\eta^{*} Y_{o}, X_{o}\right)$ of the $\mathrm{DMU}_{o}$ on 
the boundary of the MVRS technology is generally only weakly efficient. In order to test the strong efficiency of the projected DMU and identify any further nonradial improvements, we may utilize the second optimization stage. In this stage we incorporate the additional constraint $\eta=\eta^{*}$ in program (16), where $\eta^{*}$ is the optimal value of program (16), restate all inequalities (16b)-(16e) as equalities by the introduction of slack variables, and change the objective to the maximization of the sum of all slack variables (Cooper et al. 2007). As in the standard VRS technology, $\mathrm{DMU}_{o}$ is strongly efficient in technology MVRS if, in the first stage, we have $\eta^{*}=1$ and the optimal value of the second-stage model, i.e., the maximum sum of slack variables, is equal to zero. The same two-stage approach is also applicable in the case of MCRS models discussed in Section 6.3.

\subsection{The MVRS Multiplier Model}

The standard dual to program (16) has a complex structure and is shown in the proof of the next theorem. In order to facilitate interpretation, we restate the dual in an equivalent nonlinear form. In this statement, the vectors $v \in \mathbb{R}_{+}^{m}$ and $u \in \mathbb{R}_{+}^{s}$ are dual to the input and output constraints (16b)(16c) and (16d)-(16e) of the envelopment program (16), respectively. The sign-free scalars $\omega^{k}$, $k=1, \ldots, K$, are dual to the normalizing equalities (16h). The variables $p_{j}^{k}$ and $q_{j}^{k}$ are obtained by the transformation of the dual variables to constraints (16f) and (16g) defined in the proof of the next theorem.

THEOREm 3. The dual to the output-oriented program (16) is equivalently stated as follows:

$$
\begin{array}{ll}
\min & v^{\top} X_{o}+\sum_{k=1}^{K} \omega^{k} \\
\text { s.t. } & u^{\top} Y_{o}=1 \\
& \left(\sum_{i \in \mathrm{I}^{k}} v_{i} X_{j i}^{k}+\sum_{i \in \mathrm{I}^{\mathrm{S}}} v_{i}\left(p_{j}^{k} X_{j i}^{\mathrm{S}}\right)\right) \\
& -\left(\sum_{r \in \mathrm{O}^{k}} u_{r} Y_{j r}^{k}+\sum_{r \in \mathrm{O}^{\mathrm{S}}} u_{r}\left(q_{j}^{k} Y_{j r}^{\mathrm{S}}\right)\right)+\omega^{k} \geq 0, \quad \forall j, k, \\
& \sum_{k \in \mathcal{K}} p_{j}^{k}=\sum_{k \in \mathcal{K}} q_{j}^{k}=1, \quad \forall j, \\
& u, v \geq 0, p_{j}^{k}, q_{j}^{k} \geq 0, \forall j, k, \omega^{k} \text { sign free, } \forall k .
\end{array}
$$


Podinovski: Technologies with Multiple Component Processes

The multiplier model (17) attaches a variable weight $v_{i}$ to each input $i \in \mathrm{I}$ and $u_{r}$ to each output $r \in \mathrm{O}$. In particular, the weight of each shared input and output is the same for all component processes. This contrasts with the approach of Cook et al. (2000) in which each component process generally attaches a different weight to the same shared input.

Taking into account equalities (17d), we interpret variables $p_{j}^{k}$ and $q_{j}^{k}$ as the proportions in which the vectors of shared inputs and outputs $X_{j}^{\mathrm{S}}$ and $Y_{j}^{\mathrm{S}}$ of each DMU $j$ are allocated to each of its component processes $k$. The terms $p_{j}^{k} X_{j i}^{\mathrm{S}}$ and $q_{j}^{k} Y_{j r}^{\mathrm{S}}$ in constraint (17c) represent the corresponding amounts of the shared input $i$ and shared output $r$ allocated to process $k$ of DMU $j$.

In (17), the entire vectors $X_{j}^{\mathrm{S}}$ and $Y_{j}^{\mathrm{S}}$ are allocated to process $k$ of DMU $j$ in proportions $p_{j}^{k}$ and $q_{j}^{k}$, respectively. The latter proportions apply to all individual shared inputs and outputs, respectively. This contrasts with the approach of Cook et al. (2000) in which similar proportions do not depend on the DMU $j$ but are generally different for individual shared inputs and outputs.

It is now clear that the overall structure of the multiplier MVRS model (17) is similar to the standard multiplier VRS model. The obvious difference is that the inequalities (17c) are stated not for each observed DMU as in the standard VRS model, but for each component process $k$ of each observed DMU $j$. In these inequalities, the difference between the two expressions in the parentheses represents the difference between the total weighted input and the total weighted output attributable to process $k$ of the observed DMU $j$, including the allocated parts of the shared inputs and outputs. In line with the standard VRS model, each such inequality also includes a sign-free variable $\omega^{k}$, which depends on the process $k$. The meaning of variables $\omega^{k}$ is explored below.

To give an economic interpretation of the multiplier model (17), let $u$ and $v$ be the vectors of output and input prices, which are assumed to be the same for all DMUs and all component processes. Then the difference

$$
\left(\sum_{r \in \mathrm{O}^{k}} u_{r} Y_{j r}^{k}+\sum_{r \in \mathrm{O}^{\mathrm{S}}} u_{r}\left(q_{j}^{k} Y_{j r}^{\mathrm{S}}\right)\right)-\left(\sum_{i \in \mathrm{I}^{k}} v_{i} X_{j i}^{k}+\sum_{i \in \mathrm{I}^{\mathrm{S}}} v_{i}\left(p_{j}^{k} X_{j i}^{\mathrm{S}}\right)\right)
$$

is interpretable as the profit made by component $k$ of DMU $j$. 
As follows from inequalities $(17 \mathrm{c})$, for each $k$, the variable $\omega^{k}$ is greater than or equal to the profit generated by process $k$ of any DMU $j$. It is clear that, at optimality, for each $k=1, \ldots, K$, among the inequalities (17c) at least one (for some $j$ ) is satisfied as equality. (Obviously, such $j$ may be different for different $k$.) Indeed, if this were not so for some $k^{*}$, it would be possible to reduce $\omega^{k^{*}}$ and further improve the objective function, which is impossible by the assumption of optimality. Therefore, for each $k$, the optimal $\omega^{k}$ is equal to the maximum profit that component process $k$ generates across all observed DMUs.

Using (17b), we restate the objective function in (17a) as

$$
v^{\top} X_{o}+\sum_{k=1}^{K} \omega^{k}=\left(1-u^{\top} Y_{o}\right)+v^{\top} X_{o}+\sum_{k=1}^{K} \omega^{k}=1+\left[\sum_{k=1}^{K} \omega^{k}-\left(u^{\top} Y_{o}-v^{\top} X_{o}\right)\right] .
$$

Minimizing this function is equivalent to minimizing the difference between the sum of maximum profits of all component processes $\sum_{k=1}^{K} \omega^{k}$ and the actual profit generated by $\mathrm{DMU}_{o}$. Because the latter is equal to the sum of profits made by all components of $\mathrm{DMU}_{o}$, this difference is always nonnegative, and the optimal value of objective function (19) is always greater than or equal to 1 . It is now clear that $\mathrm{DMU}_{o}$ is output radial efficient, i.e., the optimal value of the multiplier model (17) is equal to 1 , if and only if there exist input and output prices $v$ and $u$, and allocation of shared inputs and outputs to component processes $p_{j}^{k}$ and $q_{j}^{k}$ such that the profit generated by each component process $k$ of $\mathrm{DMU}_{o}$ attains the maximum value $\omega^{k}$ across all observed DMUs. In simple words, $\mathrm{DMU}_{o}$ is output radial efficient if, for some prices and allocations of shared inputs and outputs, all of its component processes operate efficiently in the sense of profit maximization.

In this respect, the multiplier model (17) identifies the most favorable input and output prices $v$ and $u$, and the most favorable allocation of the shared inputs and outputs to component processes $p_{j}^{k}$ and $q_{j}^{k}$ for $\mathrm{DMU}_{o}$ under the assessment. These prices and allocations minimize the difference between the sum of the maximum profits of all individual component processes and the total profit of $\mathrm{DMU}_{o}$.

It is worth emphasizing that the multiplier model (17) is obtained by transformation of the dual of the envelopment model (16). Consequently, the following properties of the multiplier model (17) 
are not arbitrarily assumed but are a formal result of the axiomatic choices made in the development of MVRS technology: (i) the prices $v_{i}$ and $u_{r}$ of the shared inputs and outputs are the same for all DMUs and their component processes; (ii) the proportions $p_{j}^{k}$ and $q_{j}^{k}$ in which the shared inputs and outputs are allocated to each component process $k$ are generally different for each observed DMU $j$ but do not depend on the individual shared inputs and outputs; (iii) each component process $k$ is characterized by its own sign-free variable $\omega^{k}$ interpretable as the maximum profit on this process attained among all observed DMUs.

\subsection{The MCRS Models}

By Proposition 6, the output-oriented MCRS envelopment model is obtained from program (16) by removing equalities (16h). Its interpretation is similar to the MVRS model and is not given.

The dual MCRS multiplier model is obtained from program (17) by removing the sign-free variables $\omega^{k}, k=1, \ldots, K$, from the objective function and constraints:

$$
\begin{array}{ll}
\min & v^{\top} X_{o} \\
\text { s.t. } & u^{\top} Y_{o}=1 \\
& \left(\sum_{i \in \mathrm{I}^{k}} v_{i} X_{j i}^{k}+\sum_{i \in \mathrm{I}^{\mathrm{S}}} v_{i}\left(p_{j}^{k} X_{j i}^{\mathrm{S}}\right)\right) \\
& -\left(\sum_{r \in \mathrm{O}^{k}} u_{r} Y_{j r}^{k}+\sum_{r \in \mathrm{O}^{\mathrm{S}}} u_{r}\left(q_{j}^{k} Y_{j r}^{\mathrm{S}}\right)\right) \geq 0, \quad \forall j, k \\
& \sum_{k \in \mathcal{K}} p_{j}^{k}=\sum_{k \in \mathcal{K}} q_{j}^{k}=1, \quad \forall j \\
& u, v \geq 0, p_{j}^{k}, q_{j}^{k} \geq 0, \forall j, k .
\end{array}
$$

The MCRS multiplier model (20) is also interpretable similar to its MVRS analogue (17). Namely, let $v$ and $u$ be the vectors of input and output prices. These do not depend on the DMU and the component process which uses them. The model allocates all shared inputs and outputs to component processes $k=1, \ldots, K$ of each DMU $j=1, \ldots, n$ in proportions $p_{j}^{k}$ and $q_{j}^{k}$, respectively.

Inequalities (20c) mean that no component process $k$ of any DMU $j$ is allowed to make a positive profit (18). Using (20b), we can restate the objective function (20a) as follows:

$$
v^{\top} X_{o}=\left(1-u^{\top} Y_{o}\right)+v^{\top} X_{o}=1-\left(u^{\top} Y_{o}-v^{\top} X_{o}\right)
$$


According to (21), minimizing the objective function $v^{\top} X_{o}$ subject to the constraints of program (20) is equivalent to maximizing the profit $u^{\top} Y_{o}-v^{\top} X_{o}$ made by $\mathrm{DMU}_{o}$ (equal to the sum of profits made by its component processes). The following interpretation can now be given.

The MCRS multiplier model identifies the input and output prices and the allocation of shared inputs and outputs to component processes in a way that maximizes the profit made by $\mathrm{DMU}_{o}$, subject to the condition that no component process of any observed DMU can have a positive profit, and subject to the normalizing condition on the output prices (20b).

As follows from equality (21), $\mathrm{DMU}_{o}$ is output radial efficient if and only if, at optimality, it makes a zero overall profit $u^{\top} Y_{o}-v^{\top} X_{o}$. This is of course equivalent to each component process $k$ of $\mathrm{DMU}_{o}$ being efficient, i.e., making a zero profit (18). This result may be seen as an analogue of the characterization of efficiency in the standard single-process CRS technology in which a price-taking firm is efficient if and only if it makes a zero profit. As shown, in a multi-component environment, the assumption of CRS implies a zero profit for each component of an efficient firm.

\section{Technologies without Component-Specific Inputs or Outputs}

Suppose that all inputs are shared and that there are no component-specific inputs, i.e., the sets $\mathrm{I}^{k}, k=1, \ldots, K$, are empty sets. In this case, each $\operatorname{DMU}(X, Y)$ uses some (unknown to us) part of the vector of shared inputs $X^{\mathrm{S}}$ to produce the vector of component-specific outputs $Y^{k}$ and an (unknown to us) part of the vector of shared outputs $Y^{\mathrm{S}}$. Such situation arises if, for example, we use costs and labour as inputs for all processes but the data are only available in total, without allocation to the component processes.

Similarly, suppose that all outputs are shared and that there are no component-specific outputs, i.e., the sets $\mathrm{O}^{k}, k=1, \ldots, K$, are empty sets. In this case, each $\operatorname{DMU}(X, Y)$ uses its vector $X^{k}$ of component-specific inputs and some (unknown to us) part of the vector of shared inputs $X^{\mathrm{S}}$, to produce an (unknown to us) part of the vector of shared outputs $Y^{\mathrm{S}}$.

Proposition 9. Let all sets $\mathrm{I}^{k}, k=1, \ldots, K$, be empty sets. Alternatively, let all sets $\mathrm{O}^{k}, k=$ $1, \ldots, K$, be empty sets. Then the MCRS technology $T_{\mathrm{MCRS}}$ coincides with the standard CRS technology $T_{\mathrm{CRS}}$. 
Podinovski: Technologies with Multiple Component Processes

Article submitted to Operations Research; manuscript no. (Please, provide the mansucript number!)

According to Proposition 9, under the assumption of CRS, there are no benefits in considering multiple component processes unless there are both component-specific inputs and outputs.

It is worth noting that a similar result is not true under the assumption of VRS. Namely, even if all inputs or all outputs (but not both) are shared, $T_{\mathrm{VRS}}$ is generally only a subset of the multicomponent technology $T_{\text {MVRS }}$. We illustrate the difference between the two technologies by an example at the end of this section. To see practical implications of this fact, in Section 9.3, we consider an application without component-specific inputs in which the MVRS model provides a better discrimination on efficiency than the VRS model.

It should be emphasized that the advantage in the discriminating power of the MVRS model over the standard VRS model is explained by the ability of the former model to form independent convex combinations of the component processes, which is impossible in the latter model. Therefore, we can use the MVRS model only if we have a multi-component technology which satisfies Axiom 3*, and should not use this model otherwise. In particular, if all inputs are shared, it would generally be incorrect to seek additional discriminating power by declaring the production of all outputs (or groups of outputs) as separate processes, unless such separation is genuine.

Finally, assume that we have a multi-component technology but all data about the inputs and outputs is given in the aggregate form across all component processes. In this case, the sets of component-specific inputs and outputs $\mathrm{I}^{k}$ and $\mathrm{O}^{k}, k=1, \ldots, K$, are empty sets. Although we can still formally use the MVRS and MCRS technologies stated by conditions (11) and (14) or, equivalently, by (12) and (15), the resulting technologies coincide with the standard VRS and CRS technologies, respectively. In other words, in the described scenario, there are no benefits of treating the technology as consisting of multiple components.

Proposition 10. Let all sets $\mathrm{I}^{k}$ and $\mathrm{O}^{k}, k=1, \ldots, K$, be empty sets (but the sets $X^{\mathrm{S}}$ and $Y^{\mathrm{S}}$ be not empty). Then $T_{\mathrm{MVRS}}=T_{\mathrm{VRS}}$ and $T_{\mathrm{MCRS}}=T_{\mathrm{CRS}}$.

EXAmPLE 2. Consider the MVRS and MCRS technologies generated by the DMUs $A$ and $B$ shown in Table 2. These DMUs use a a single shared input $X^{\mathrm{S}}$ to produce two component-specific outputs 
Table 2 DMUs in Example 2

\begin{tabular}{lccc}
\hline DMU & $X^{\mathrm{S}}$ & $Y^{1}$ & $Y^{2}$ \\
\hline$A$ & 1 & 2 & 1 \\
$B$ & 1 & 1 & 2 \\
$C: \lambda^{1}=(0.5,0.5), \lambda^{2}=(0,1)$ & 1.5 & 1.5 & 2 \\
$D: \lambda^{1}=(1,0), \lambda^{2}=(0,1)$ & 2 & 2 & 2 \\
\hline
\end{tabular}

$Y^{1}$ and $Y^{2}$. Note that there are no component-specific inputs and there are no shared outputs.

Consider two further hypothetical DMUs $C$ and $D$. Both are obtained by combining component processes of DMUs $A$ and $B$ using formula (7) in which the vectors $\lambda^{1}=\left(\lambda_{A}^{1}, \lambda_{B}^{1}\right)$ and $\lambda^{2}=\left(\lambda_{A}^{2}, \lambda_{B}^{2}\right)$ are as shown in Table 2. Therefore, DMUs $C$ and $D$ are included in both technologies $T_{\text {MvRS }}$ and $T_{\mathrm{MCRS}}$. (In particular, both satisfy conditions (11) with the specified vectors $\lambda^{1}$ and $\lambda^{2}$.)

In line with Proposition 9, DMUs $C$ and $D$ are also included in the standard CRS technology. To verify this independently, we may for example note that DMU $D$ is weakly dominated by the DMU $A^{*}=(2,4,2)$ obtained by scaling DMU $A$ by a factor of $\rho=2$ as in Axiom 4 .

However, as is easy to verify, neither DMU $C$ nor $D$ is in the standard VRS technology. This shows that the statement of Proposition 9 cannot be extended to the case of the MVRS technology.

\section{Scale Characteristics of the MVRS Technology}

The notions of optimal scale, scale efficiency, scale elasticity and returns to scale are important characteristics of production frontiers. For the standard VRS technology and, more generally, for the large classes of convex and, in particular, polyhedral technologies, all these characteristics have been extensively studied in the literature. In this section, we rely on these results and give a cursory overview of scale characteristics in the MVRS technology, while pointing out some nontrivial issues.

\subsection{The Closed Cone Extension of the MVRS Technology}

As shown by Banker (1984), the notions of the most productive scale size (MPSS) and scale efficiency in the standard VRS technology $T_{\mathrm{VRS}}$ can be expressed in terms of the closed cone extension of technology $T_{\mathrm{VRS}}$, which coincides with the CRS technology of Charnes et al. (1978). 
It turns out that, in the evaluation of scale efficiency of DMUs in the MVRS technology, its closed cone extension plays a similar role.

Let us first define the convex cone $C$ generated by technology $T_{\mathrm{MVRS}}$ as the set of all DMUs located on the rays from the origin through the points in the technology:

$$
C=\left\{(X, Y) \in \mathbb{R}^{m+s} \mid \exists\left(X^{\prime}, Y^{\prime}\right) \in T_{\mathrm{MVRS}}, \alpha \geq 0:(X, Y)=\left(\alpha X^{\prime}, \alpha Y^{\prime}\right)\right\}
$$

Because the cone $C$ may not be closed (see Example 3 below which illustrates this possibility for the standard VRS technology as a special case of the MVRS technology), we define the closed cone $\bar{C}$ as the closure of $C$ :

$$
\bar{C}=\operatorname{cl} C
$$

As follows from the general result established by Podinovski and Bouzdine-Chameeva (2019, Proposition 3), the closed cone extension $\overline{\mathcal{C}}$ is defined by the same statements (11) or (12) as technology $T_{\text {MVRS }}$, with the following modification. In both statements, we introduce a new variable $\alpha \geq 0$ and replace the normalizing conditions (11e) and (12g) by the following equalities:

$$
1^{\top} \lambda^{k}=\alpha, \quad \forall k \in \mathcal{K}
$$

It is clear that the closed cone extension $\overline{\mathcal{C}}$ is a subset of technology $T_{\text {MCRS }}$ and is generally not equal to it. Indeed, conditions (24) restrict the scaling of all individual processes in the cone $\overline{\mathcal{C}}$ by the same scaling parameter $\alpha$. In contrast, the individual component processes in the MCRS technology can be scaled by generally different scaling factors. This follows from Axiom $4^{*}$ and can also be seen from the fact that the normalizing conditions (11e) used in the statement of the MVRS technology are completely omitted in the statements (14) and (15) of the MCRS technology.

It is also straightforward to verify that the standard CRS technology $T_{\mathrm{CRS}}$ is a subset of the closed cone $\overline{\mathcal{C}}$ and is generally different from the latter. Therefore, we always have the following two embeddings which cannot generally be changed to equalities:

$$
T_{\mathrm{CRS}} \subseteq \bar{C} \subseteq T_{\mathrm{MCRS}}
$$


Example 3. Let us give an example in which the cone $C$ defined by (22) is not a closed set. Consider the VRS technology as a special case of the MVRS technology with a single component process, generated by the single DMU $A=(1,1,1)$, where the first two components are inputs and the last component is output. It is straightforward to verify that the DMU $B=(1,0,0)$ is not in the cone $C$ but is in its closure $\bar{C}$ which coincides with the CRS technology generated by $A$. Therefore, $C \neq \bar{C}$ and $C$ is not a closed set.

\subsection{Scale Efficiency}

The definition of MPSS given by Banker (1984) for the standard VRS technology generalizes the earlier definition of optimal scale of Frisch (1965). As shown by Podinovski (2017), the notion of MPSS and methods of its evaluation can be extended to any convex technology.

Consider any DMU $\left(X_{o}, Y_{o}\right) \in T_{\mathrm{MVRS}}$ such that $Y_{o} \neq 0$. In line with the approach of Banker (1984), consider the following program:

$$
\begin{aligned}
& \theta^{*}=\sup (\beta / \alpha) \\
& \text { subject to }\left(\alpha X_{o}, \beta Y_{o}\right) \in T_{\mathrm{MVRS}}, \alpha>0, \beta \geq 0 .
\end{aligned}
$$

Because $\alpha=\beta=1$ is a feasible solution of program (25), we always have $\theta^{*} \geq 1$. Following the definition of Banker (1984), DMU $\left(X_{o}, Y_{o}\right)$ is said to be at MPSS if $\theta^{*}=1$. In this case, there exists no DMU $\left(\alpha X_{o}, \beta Y_{o}\right) \in T_{\text {MVRS }}$ whose average productivity (measured as the ratio of the quantity of output $\beta$ to the quantity of input $\alpha)$ is higher than the average productivity of $\operatorname{DMU}\left(X_{o}, Y_{o}\right)$, equal to 1.

Let us now refer to the standard VRS technology which is a special case of the MVRS technology. As shown by Banker (1984), in this case the optimal value $\theta^{*}$ of program (25) is equal to the inverse of the input (or, equivalently, output) radial efficiency of DMU $\left(X_{o}, Y_{o}\right)$ in the closed cone extension of the VRS technology, which coincides with the standard CRS technology of Charnes et al. (1978). Podinovski and Bouzdine-Chameeva (2019) prove that a similar result is true for any polyhedral technology, including the MVRS technology $T_{\mathrm{MVRS}}$. It follows that the evaluation of 
Podinovski: Technologies with Multiple Component Processes

$\theta^{*}$ by solving program (25) is equivalent to the evaluation of the output radial efficiency of DMU $\left(X_{o}, Y_{o}\right)$ in the closed cone extension $\overline{\mathcal{C}}$ of technology $T_{\text {MVRS }}$ defined by $(23)$ :

$$
\begin{aligned}
& \theta^{*}=\max \theta \\
& \text { subject to }\left(X_{o}, \theta Y_{o}\right) \in \bar{C}, \theta \geq 0 .
\end{aligned}
$$

It is worth noting that program (26) is formally obtained from program (25), without any assumptions that the cone $\bar{C}$ represents a production technology. Rephrasing Ray (2004, pages 56 and 61) who made a similar argument in the case of the standard VRS technology, we make no assumption that DMUs in the cone $\bar{C}$ are producible. The latter is just a mathematical construct formally arising in the process of linearization of program (25).

Similar to the case of the standard VRS technology, we can now consider the notion of overall (or aggregate) efficiency of DMU $\left(X_{o}, Y_{o}\right) \in T_{\mathrm{MVRS}}$ and its decomposition into the technical and scale components.

Following Banker (1984) and Banker et al. (1984), we define the overall efficiency $O E\left(X_{o}, Y_{o}\right)$ of the $\operatorname{DMU}\left(X_{o}, Y_{o}\right)$ as the inverse $1 / \theta^{*}$ of the optimal value of either program (25) or (26). We also define the technical efficiency $T E\left(X_{o}, Y_{o}\right)$ of the DMU $\left(X_{o}, Y_{o}\right)$ as its output radial efficiency in technology $T_{\text {MVRS }}$ equal to the inverse $1 / \eta^{*}$ of the optimal value of program (16). This leads to the conventional decomposition:

$$
O E\left(X_{o}, Y_{o}\right)=T E\left(X_{o}, Y_{o}\right) \times S E\left(X_{o}, Y_{o}\right)
$$

where $\operatorname{SE}\left(X_{o}, Y_{o}\right)=O E\left(X_{o}, Y_{o}\right) / T E\left(X_{o}, Y_{o}\right) \leq 1$ is the scale efficiency of DMU $\left(X_{o}, Y_{o}\right)$.

To see the meaning of scale efficiency $S E\left(X_{o}, Y_{o}\right)$, suppose that DMU $\left(X_{o}, Y_{o}\right)$ is technically efficient, i.e., let $T E\left(X_{o}, Y_{o}\right)=1$. Then $O E\left(X_{o}, Y_{o}\right)=S E\left(X_{o}, Y_{o}\right)$ and any inefficiency of DMU $\left(X_{o}, Y_{o}\right)$ is attributable to its suboptimal scale. Reinterpreting Banker (1984), the scale efficiency $S E\left(X_{o}, Y_{o}\right)$ can be viewed as a measure of divergence of DMU $\left(X_{o}, Y_{o}\right)$ from MPSS. As seen from program (25), $S E\left(X_{o}, Y_{o}\right)$ is equal to the factor $\theta^{*}$ by which the average productivity $\beta / \alpha=1$ of DMU $\left(X_{o}, Y_{o}\right)$ could be improved if it were to move to MPSS by changing the scale of production. 


\subsection{Scale Elasticity and Returns to Scale}

Banker and Thrall (1992) explored the notion of returns-to-scale (RTS) in the standard VRS technology based on the one-sided (left-hand and right-hand) scale elasticity evaluated at any efficient $\mathrm{DMU}_{o}$. The literature on the topic of RTS for VRS production frontiers has since grown significantly—see, e.g., Fukuyama (2000), Førsund and Hjalmarsson (2004), Hadjicostas and Soteriou (2006), Chambers and Färe (2008), Podinovski and Førsund (2010), Sahoo and Tone (2015), and Zelenyuk (2013). As further shown by Podinovski (2017), the scale elasticity and RTS are correctly defined in any convex technology, and not only in the standard VRS technology. Because technology $T_{\text {MVRS }}$ is convex, both notions are conceptually applicable to it as well, and a question arises as to how to evaluate these characteristics.

Note that, by Proposition $3, T_{\mathrm{MVRS}}$ is a polyhedral set. This allows us to use the approach to the evaluation of one-sided scale elasticities and RTS developed for any polyhedral technology by Podinovski et al. (2016). Namely, let DMU ${ }_{o}$ be output radial efficient. (The case of inefficient DMUs is conventionally resolved by evaluating RTS at their chosen projections on the production frontier.) Denote $\omega^{\text {min }}$ and $\omega^{\text {max }}$ the minimum and maximum of the sums $\sum_{k=1}^{K} \omega^{k}$, respectively, taken over the set of all optimal solutions to the multiplier program (17), or to its linear programming analogue stated in the proof of Theorem 3. Similar to the case of the standard VRS model in which we formally have $K=1$, the evaluation of $\omega^{\min }$ and $\omega^{\max }$ requires solving two linear programs.

Denote $\varepsilon^{-}\left(X_{o}, Y_{o}\right)$ and $\varepsilon^{+}\left(X_{o}, Y_{o}\right)$ the left-hand and right-hand scale elasticities evaluated at $\mathrm{DMU}_{o}$, respectively. Applying the general result proved by Podinovski et al. (2016, page 241) to technology $T_{\mathrm{MVRS}}$, we have:

$$
\varepsilon^{-}\left(X_{o}, Y_{o}\right)=1-\omega^{\min }, \quad \varepsilon^{+}\left(X_{o}, Y_{o}\right)=1-\omega^{\max } .
$$

It is clear that we always have $\varepsilon^{-}\left(X_{o}, Y_{o}\right) \geq \varepsilon^{+}\left(X_{o}, Y_{o}\right)$. We also conventionally allow the case $\omega^{\text {min }}=-\infty$ or, equivalently, $\varepsilon^{-}\left(X_{o}, Y_{o}\right)=+\infty$, which corresponds to the "vertical" weakly efficient facets of the technology. 
Podinovski: Technologies with Multiple Component Processes

Article submitted to Operations Research; manuscript no. (Please, provide the mansucript number!)

The characterization of RTS based on the one-sided scale elasticities (27) is now straightforward and is aligned with the definition of Banker and Thrall (1992). Namely, an output radial efficient $\mathrm{DMU}_{o}$ exhibits increasing returns to scale (IRS) if $\varepsilon^{+}\left(X_{o}, Y_{o}\right)>1$, decreasing returns to scale (DRS) if $\varepsilon^{-}\left(X_{o}, Y_{o}\right)<1$, and constant returns to scale (CRS) otherwise, i.e., if $\varepsilon^{+}\left(X_{o}, Y_{o}\right) \leq 1 \leq \varepsilon^{-}\left(X_{o}, Y_{o}\right)$.

As shown by Banker and Thrall (1992), in the standard VRS technology, a technically efficient $\operatorname{DMU}\left(X_{o}, Y_{o}\right)$ exhibits CRS if and only if it is at MPSS. Podinovski (2017) proves this result for any convex technology. It follows that a DMU $\left(X_{o}, Y_{o}\right) \in T_{\text {MVRS }}$ exhibits CRS if and only if it is at MPSS, which in turn is equivalent to the output radial efficiency of DMU $\left(X_{o}, Y_{o}\right)$ in the closed cone $\bar{C}$ evaluated by program (26) being equal to 1 .

REMARK 5. Similar to the case of standard VRS technology, the RTS characterization in the MVRS technology can also be given using the input-oriented MVRS multiplier model. Let $\tilde{\omega}^{k}$, $k \in \mathcal{K}$, be the dual variables to the normalizing equalities (16h) in the input-oriented analogue of model (16). Define $\tilde{\omega}^{\min }$ and $\tilde{\omega}^{\max }$ the minimum and maximum of the sums $\sum_{k=1}^{K} \tilde{\omega}^{k}$, respectively, taken over the set of all optimal solutions to the input-oriented multiplier program. According to the general result applicable to any polyhedral technology established by Podinovski et al. (2016, page 249), if $\mathrm{DMU}_{o}$ is both input and output radial efficient, then we have $\varepsilon^{-}\left(X_{o}, Y_{o}\right)=1 /\left(1-\tilde{\omega}^{\max }\right)$ and $\varepsilon^{+}\left(X_{o}, Y_{o}\right)=1 /\left(1-\tilde{\omega}^{\text {min }}\right)$. (If $\mathrm{DMU}_{o}$ is not input radial efficient, this approach will evaluate the one-sided scale elasticities at the input radial projection of $\mathrm{DMU}_{\circ}$.)

\section{Application}

In this section, we present an illustrative application of the MVRS and MCRS models to the efficiency assessment of public universities in England. We use a combination of open data from the UK Higher Education Statistics Agency (HESA) available at www.hesa.ac.uk and data from the bibliographic database Scopus.

\subsection{Data and Model Specification}

We limit this application to all public universities in England that provide a range of undergraduate and postgraduate programs across different areas of study. For homogeneity reasons, we exclude 
specialized providers of medical education and several colleges of creative arts. We also exclude providers that offer postgraduate education only. Our final sample consists of 102 universities.

Instead of viewing each university as a single production process, we consider it as a combination of three component processes. The first comprises departments of medicine (including dentistry), the second includes science and engineering, and the third includes non-science departments such as social sciences, humanities and arts. Although not perfect, this separation acknowledges the different approaches to teaching and research, and the associated cost structures between the different disciplines.

Table 3 shows the inputs and outputs used in this application. The first three inputs are component-specific and represent the total expenditure by the three groups of department. Each such input is a combination of academic and non-academic staff costs and the other expenses at the departmental level. Input 4 represents centralized expenditure at the university level on academic and non-academic staff and various services. Because we do not know the allocation of this expenditure to the departments, it is considered to be a shared input.

On the output side, the first six measures are component-specific and represent the number of undergraduate (UG) and postgraduate (PG) students at each of the three groups of department. The last output 7 represents the number of papers published by all academic staff of the university. This measure was obtained by searching the Scopus database by the university affiliation and is considered to be a shared output whose exact allocation to the departments is unknown.

The data for all inputs and outputs (except output 7) is as recorded by HESA in 2018/19. Output 7 represents the papers with the publication date in 2019.

\subsection{Computational Results}

We evaluate the efficiency of universities by solving the output-oriented MVRS envelopment program (16) and its MCRS analogue obtained from (16) by removing the normalizing equalities (16h). For comparison purposes, we also solve the standard output-oriented VRS and CRS models defined by the same set of four inputs and seven outputs. 
Podinovski: Technologies with Multiple Component Processes

Article submitted to Operations Research; manuscript no. (Please, provide the mansucript number!)

Table 3 Descriptive statistics for the data used in the application

Note: All expenditures (Inputs 1-4) are in million British pounds.

\begin{tabular}{lrrrrr}
\hline & & & & & $\begin{array}{r}\text { Standard } \\
\text { deviation }\end{array}$ \\
\hline Measure & Mean & Median & Minimum & Maximum \\
Component-specific inputs: expenditure & & & & & \\
Input 1: Medical departments & 12.43 & 0 & 0 & 130.87 & 28.42 \\
Input 2: Science departments & 46.17 & 37.49 & 0 & 210.1 & 42.43 \\
Input 3: Non-science departments & 51.05 & 42.54 & 0 & 221.1 & 38.17 \\
Shared input & & & & & \\
Input 4: Centralized expenditure & 230.23 & 129.75 & 10.36 & 2058.55 & 317.87 \\
Component-specific outputs: students & & & & & \\
Output 1: UG medical students & 352.21 & 0 & 0 & 2945 & 699.4 \\
Output 2: UG science students & 5493.04 & 5192.5 & 180 & 44190 & 4858.57 \\
Output 3: UG non-science students & 7365.44 & 6530 & 50 & 55745 & 5985.65 \\
Output 4: PG medical students & 145.83 & 0 & 0 & 2310 & 387.59 \\
Output 5: PG science students & 1690.05 & 1475 & 0 & 7590 & 1387.77 \\
Output 6: PG non-science students & 2687.84 & 2245 & 20 & 11275 & 1911.8 \\
Shared output & & & & & \\
Output 7: Published papers & 1933.87 & 772 & 0 & 16364 & 3067.31 \\
\hline
\end{tabular}

Table 4 Output radial efficiency in different models

\begin{tabular}{lcccc}
\hline Model & $\begin{array}{l}\text { Number of } \\
\text { efficient DMUs }\end{array}$ & $\begin{array}{l}\text { Average } \\
\text { efficiency }\end{array}$ & $\begin{array}{l}\text { Minimum } \\
\text { efficiency }\end{array}$ & $\begin{array}{l}\text { Standard } \\
\text { deviation }\end{array}$ \\
\hline VRS & 64 & 0.951 & 0.547 & 0.0862 \\
MVRS & 62 & 0.9484 & 0.547 & 0.0873 \\
CRS & 50 & 0.9263 & 0.5316 & 0.1024 \\
MCRS & 44 & 0.9168 & 0.4865 & 0.1091 \\
\hline
\end{tabular}

Table 5 Differences between the four models

\begin{tabular}{lcccc}
\hline Models & $\begin{array}{l}\text { Number of DMUs } \\
\text { with different } \\
\text { efficiency scores }\end{array}$ & $\begin{array}{l}\text { Maximum } \\
\text { difference } \\
\text { in efficiency }\end{array}$ & $\begin{array}{l}\text { Average } \\
\text { difference } \\
\text { in efficiency }\end{array}$ & $\begin{array}{l}\text { Average difference } \\
\text { if efficiency } \\
\text { is different }\end{array}$ \\
\hline VRS-MVRS & 16 & 0.0948 & 0.0026 & 0.0167 \\
CRS-MCRS & 32 & 0.2737 & 0.0094 & 0.0301 \\
VRS-CRS & 52 & 0.3928 & 0.0248 & 0.0486 \\
MVRS-MCRS & 58 & 0.5135 & 0.0316 & 0.0555 \\
\hline
\end{tabular}


Table 4 shows summary statistics for the results obtained by solving all four models (all values in this and the following tables are rounded). Because technology $T_{\mathrm{VRS}}$ is a subset of technology $T_{\mathrm{MVRS}}$, and $T_{\mathrm{CRS}}$ is a subset of $T_{\mathrm{MCRS}}$, the MVRS and MVRS models provide a better discrimination on efficiency than the standard VRS and CRS models, respectively.

Table 5 provides further details. The row "VRS-MVRS" compares the VRS and MVRS models. Its second column shows the number of DMUs whose output radial efficiency is different if assessed by the VRS and MVRS models (i.e., is higher in the former model than in the latter). The next two columns show the maximum and average differences between the efficiency scores obtained in the two models. The last column shows the average difference accounting only for those DMUs whose efficiency in the two models is different. The other three rows of Table 5 show similar statistics demonstrating the differences between the CRS and MCRS, VRS and CRS, and MVRS and MCRS models.

Several observations are worth highlighting. As expected, the use of the MVRS and MCRS models reduces the number of efficient DMUs and identifies additional inefficiencies, compared to the standard VRS and CRS models. As seen from Table 5, the MVRS model identifies additional inefficiency in 16 DMUs, which includes the 2 DMUs efficient in the VRS model. On average, this additional inefficiency is equal to 0.0167 , with its maximum equal to 0.0948. Furthermore, the MCRS model identifies additional inefficiency (compared to the CRS model) in 32 DMUs, with the average additional inefficiency equal to 0.0301 and the maximum equal to 0.2737 .

The last two rows of Table 5 show that the difference between the discriminating powers of the MCRS and MVRS models is broadly similar to the difference observed between the standard CRS and VRS models, with a slightly more pronounced difference exhibited by the pair of multicomponent models.

We now turn to the evaluation of RTS in the MVRS and VRS technologies. For comparison purposes, we consider the 62 DMUs that are output radial efficient in both MVRS and VRS models. We first evaluate the left-hand and right-hand scale elasticities $\varepsilon^{-}\left(X_{o}, Y_{o}\right)$ and $\varepsilon^{+}\left(X_{o}, Y_{o}\right)$ at each of the efficient DMUs. For the VRS technology, this is a standard procedure (see, e.g., the references 
Podinovski: Technologies with Multiple Component Processes

given in Section 8.3), and for the MVRS technology, we follow the linear programming approach described in Section 8.3. In both VRS and MVRS technologies, the type of RTS exhibited by a DMU is determined by the range between its two one-sided scale elasticities.

Computations show that, in the standard VRS technology, 2 universities exhibit IRS, 49 exhibit CRS (and are therefore at MPSS ) and 11 exhibit DRS. In the larger MVRS technology, two universities change their type from CRS to DRS. As a result, in the MVRS technology, 2 universities exhibit IRS, 47 exhibit CRS (and are at MPSS) and 13 exhibit DRS.

Although the RTS characterization of most of the efficient DMUs remains the same in both technologies, the underlying one-sided scale elasticities which characterize the strength of RTS characterization are different for 43 out of the 62 universities. For example, for one of the DMUs $\left(X_{o}, Y_{o}\right)$ whose characterization is different in the two technologies, we have $\varepsilon^{+}\left(X_{o}, Y_{o}\right)=0.0126$ and $\varepsilon^{-}\left(X_{o}, Y_{o}\right)=1.0634$ in the VRS technology, and $\varepsilon^{+}\left(X_{o}, Y_{o}\right)=0.4712$ and $\varepsilon^{-}\left(X_{o}, Y_{o}\right)=0.8681$ in the MVRS technology. The former means that this DMU exhibits CRS and is at MPSS in the VRS technology. However, it exhibits DRS and is larger than its MPSS in the MVRS technology.

These empirical results are consistent with a theoretical result recently established by Podinovski and Bouzdine-Chameeva (2020). Namely, if a DMU is output radial efficient in two technologies one of which is a subset of the other (as, for example, in our application in which the VRS technology is a subset of the MVRS technology), the range between the one-sided scale elasticities evaluated at this DMU in the larger technology is tighter than (is a subset of) the range evaluated in the smaller technology. This implies that the RTS characterization in the larger technology is more precisely defined (judging by the tighter range between the one-sided scale elasticities) than in the smaller technology.

Overall, as shown by this application, in a multi-component production setting, the MVRS and MCRS models provide useful and meaningful advantages over the standard VRS and CRS models in relation to the discriminating power on efficiency and RTS characterization. Importantly, these advantages are realized without any information about the allocation mechanism of the shared inputs and outputs. 
Table 6 Output radial efficiency in different models in the scenario with aggregated expenditures

\begin{tabular}{lcccc}
\hline Model & $\begin{array}{l}\text { Number of } \\
\text { efficient DMUs }\end{array}$ & $\begin{array}{l}\text { Average } \\
\text { efficiency }\end{array}$ & $\begin{array}{l}\text { Minimum } \\
\text { efficiency }\end{array}$ & $\begin{array}{c}\text { Standard } \\
\text { deviation }\end{array}$ \\
\hline VRS & 20 & 0.7672 & 0.3581 & 0.1728 \\
MVRS & 20 & 0.7658 & 0.3581 & 0.172 \\
CRS and MCRS & 13 & 0.6637 & 0.3392 & 0.177 \\
\hline
\end{tabular}

Table 7 Differences between the models in the scenario with aggregated expenditures

\begin{tabular}{lcccc}
\hline Models & $\begin{array}{l}\text { Number of DMUs } \\
\text { with different } \\
\text { efficiency scores }\end{array}$ & $\begin{array}{l}\text { Maximum } \\
\text { difference } \\
\text { in efficiency }\end{array}$ & $\begin{array}{l}\text { Average } \\
\text { difference } \\
\text { in efficiency }\end{array}$ & $\begin{array}{l}\text { Average difference } \\
\text { if efficiency } \\
\text { is different }\end{array}$ \\
\hline VRS-MVRS & 7 & 0.0414 & 0.0014 & 0.0207 \\
VRS-CRS & 89 & 0.497 & 0.1036 & 0.1187 \\
MVRS-MCRS & 89 & 0.497 & 0.1022 & 0.1171 \\
\hline
\end{tabular}

\subsection{Scenario with Aggregated Costs}

Let us now consider a scenario in which all expenditures of the universities are available only in aggregate, which is similar to the model setup used by Thanassoulis et al. (2011). We simulate this possibility by replacing the four inputs in Table 3 by their total. We treat the resulting total expenditure as a single shared input whose allocation to the three component processes is unknown. This creates a situation considered in Section 7 in which there are no component-specific inputs but there are component-specific (and possibly shared) outputs.

Computations show that all four (VRS, CRS, MVRS and MCRS) models provide significantly better discrimination on efficiency than in the main scenario with four separate inputs - see Table 6 . This is expected taking into account the reduced number of inputs in the new scenario. In line with Proposition 9, the CRS and MCRS models provide identical results shown in the last row of Table 6.

Similar to the main scenario discussed in Section 9.2, Table 7 highlights further differences between the models. In particular, according to Table 6 , in the considered application the VRS and MVRS models identify the same 20 efficient and 102-20=82 inefficient DMUs. However, according to Table 7, the MVRS model identifies additional inefficiencies in 7 out of the 82 inefficient DMUs, 
Podinovski: Technologies with Multiple Component Processes

with the average additional inefficiency equal to 0.0207 and the maximum equal to 0.0414 .

The last two rows of Table 7 show the difference between the VRS and CRS models and their multi-component analogues. Because the MVRS model provides a better discrimination on efficiency than the VRS model and, by Proposition 9, the MCRS and CRS models provide identical efficiency scores, the average difference of 0.1036 in efficiency between the VRS and CRS models is larger than the average difference of 0.1022 between the results of the MVRS and MCRS models.

In summary, this example shows that, even if all inputs are available only in aggregate, we may still gain in efficiency discrimination compared to the standard VRS model, by utilizing the information that the technology consists of multiple component processes and using an appropriately specified MVRS model (16).

Finally, we additionally aggregate all UG students and (separately) all PG students across all departments. The model now consists of a single expenditure treated as a single shared input and UG, PG students and publications as the three shared outputs. In line with Proposition 10, the results obtained by solving the MVRS and MCRS models are identical to the results obtained by solving the standard VRS and CRS models, respectively. This confirms that, if all inputs and outputs are shared, we do not gain any advantages by introducing component processes in the model of technology.

\section{Conclusion}

In this paper we develop new VRS and CRS models of production technologies with multiple component processes. Using the standard VRS and CRS technologies in such cases is of course possible but these models are too small as they do not allow independent convex combinations within individual component processes and, in the case of CRS, their individual scaling.

As the literature shows, developing models of technologies accounting for multiple component processes is generally a problematic task. The main difficulty here is the treatment of the inputs and outputs that are shared, in generally unknown proportions, by different component processes. In this paper, we overcome this difficulty and show that, even if the exact allocation of the shared 
inputs and outputs to component processes is unknown, we can still use its most conservative, or worst-case, estimates, and obtain a useful extension of the conventional VRS and CRS technologies. It turns out that just the information that there are several component processes, without any knowledge of the allocation mechanism for the shared inputs and outputs, may be used to construct a larger technology than the conventional models.

We start by introducing the new axioms of convexity and scalability for multi-component technologies. The multi-component VRS and CRS (referred to as MVRS and MCRS) technologies are then formally derived from the stated axioms. It is important to emphasize that no allocation mechanism for the shared inputs and outputs is used in this development. We then consider the envelopment and dual multiplier DEA models based on the MVRS and MCRS technologies.

It is interesting to note that the MVRS and MCRS multiplier models exhibit a particular allocation mechanism for the shared inputs and outputs that favors the DMU under the assessment, and that this mechanism is different from the approach already explored in the literature, in several important respects. In our case, this allocation mechanism is not postulated but is a formal consequence of the production axioms that do not assume any particular allocation.

To complement the development of the MVRS and MCRS technologies and the envelopment and multiplier models based on them, we also explore a range of scale characteristics of the MVRS production frontier. We show that the notions of most productive scale size, scale efficiency, scale elasticity and returns to scale arising in the MVRS technology are all natural generalizations of the corresponding standard notions in the VRS model.

To outline possible research extensions to this paper, let us note that many established production technologies developed in the DEA literature are based on the implicit generic assumption that all inputs are used in the production of all outputs. This assumption is reflected in the standard axioms of convexity and scalability that form a part of their axiomatic foundations and allow only the whole DMUs to be combined or scaled, without a possibility of component-specific transformations. The approach developed in this paper could be used for the restatement of many existing technologies for the case of multiple component processes. 


\section{Appendix A. Proofs}

Proof of Proposition 1. Consider any $i \in \mathrm{I}^{\mathrm{S}}$. Rearranging equality (9b), we have:

$$
\hat{X}_{i}^{\mathrm{S}}=\sum_{l=1}^{L}\left(\sum_{k=1}^{K} \lambda_{l}^{k} \alpha_{l i}^{k}\right) \tilde{X}_{l i}^{\mathrm{S}} .
$$

For each $l$, we have $\sum_{k=1}^{K} \lambda_{l}^{k} \alpha_{l i}^{k} \leq \max _{k \in \mathcal{K}}\left\{\lambda_{l}^{k}\right\}$. Let the maximum on the right-hand side be attained at $k=k^{*}$. The stated inequality becomes equality at the vector $\alpha_{l i}=\left(\alpha_{l i}^{1}, \ldots, \alpha_{l i}^{K}\right)$ where $\alpha_{l i}^{k^{*}}=1$ and $\alpha_{l i}^{k}=0$ if $k \neq k^{*}$. The proof for the shared outputs $r \in \mathrm{O}^{\mathrm{S}}$ is similar.

Proof of Theorem 1. Denote $T^{*}$ the set of all DMUs $(X, Y) \geq 0$ that satisfy (11) with some vectors $\lambda^{k} \in \mathbb{R}^{n}, k \in \mathcal{K}$. We need to prove that $T_{\text {MVRS }}=T^{*}$. Let $(X, Y) \in T^{*}$. Then $(X, Y)$ satisfies $(11)$

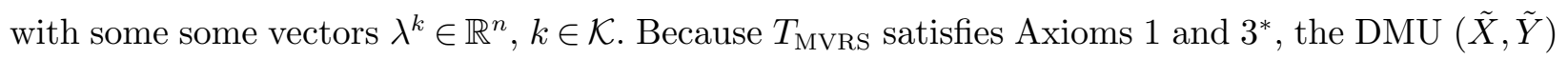
on the left-hand side of inequalities (11a)-(11d) is in $T_{\text {Mvrs }}$. By Axiom 2, the DMU $(X, Y)$, which appears on the right-hand side of the same inequalities, is in $T_{\mathrm{MVRS}}$. Therefore, $T^{*} \subseteq T_{\mathrm{MVRS}}$. By Lemma 1 proved below, $T^{*}$ satisfies Axioms 1,2 and $3^{*}$. Because, by definition, $T_{\mathrm{MVRS}}$ is the smallest technology that satisfies these axioms, we have $T_{\mathrm{MVRS}} \subseteq T^{*}$. Therefore, $T_{\mathrm{MVRS}}=T^{*}$.

Lemma 1. Technology $T^{*}$ defined in the statement of Theorem 1 satisfies Axioms 1, 2 and 3*.

Proof of Lemma 1. Any observed DMU $\left(X_{j^{*}}, Y_{j^{*}}\right), j^{*} \in J$, satisfies (11) with $\lambda_{j}^{k}$ defined as follows: for all $k \in \mathcal{K}, \lambda_{j^{*}}^{k}=1$ and $\lambda_{j}^{k}=0$ if $\neq j^{*}$. Therefore, $T^{*}$ satisfies Axiom 1. Consider Axiom 2. Let $(X, Y)$ satisfy (11) with some vectors $\lambda^{k} \in \mathbb{R}^{n}, k \in \mathcal{K}$. Then $\left(X^{\prime}, Y^{\prime}\right)$ satisfies (11) with the same $\lambda^{k}, k \in \mathcal{K}$. Therefore, $T^{*}$ satisfies Axiom 2.

To prove that $T^{*}$ satisfies Axiom $3^{*}$, assume that all $L$ DMUs (8) are members of $T^{*}$. We need to prove that, for any $K$ vectors $\lambda^{k} \in \mathbb{R}_{+}^{L}$ such that $1^{\top} \lambda^{k}=1$, for all $k \in \mathcal{K}$, we have $(\tilde{X}, \tilde{Y}) \in T^{*}$, where $(\tilde{X}, \tilde{Y})$ is defined by $(10)$. Each DMU $\left(X_{l}, Y_{l}\right), l=1, \ldots, L$, satisfies (11) with some vectors $\tilde{\lambda}_{j}^{k}(l)$ specific to this DMU. Consider (10). Taking into account (11b), for each $k \in \mathcal{K}$, we have:

$$
\tilde{X}^{k}=\sum_{l=1}^{L} \lambda_{l}^{k} X_{l}^{k} \geq \sum_{l=1}^{L}\left(\lambda_{l}^{k} \sum_{j=1}^{n} \tilde{\lambda}_{j}^{k}(l) X_{j}^{k}\right)=\sum_{j=1}^{n} \hat{\lambda}_{j}^{k} X_{j}^{k},
$$

where

$$
\hat{\lambda}_{j}^{k}=\sum_{l=1}^{L} \lambda_{l}^{k} \tilde{\lambda}_{j}^{k}(l)
$$


Note that $\hat{\lambda}^{k}=\left(\hat{\lambda}_{1}^{k}, \ldots, \hat{\lambda}_{n}^{k}\right) \geq 0$ and $1^{\top} \hat{\lambda}^{k}=1$, for all $k \in \mathcal{K}$. Furthermore,

$$
\begin{aligned}
\tilde{X}^{\mathrm{S}} & =\sum_{l=1}^{L} \max _{k \in \mathcal{K}}\left\{\lambda_{l}^{k}\right\} X_{l}^{\mathrm{S}} \\
& \geq \sum_{l=1}^{L}\left(\max _{k \in \mathcal{K}}\left\{\lambda_{l}^{k}\right\} \sum_{j=1}^{n} \max _{k \in \mathcal{K}}\left\{\tilde{\lambda}_{j}^{k}(l)\right\} X_{j}^{\mathrm{S}}\right) \\
& \geq \sum_{j=1}^{n}\left(\max _{k \in \mathcal{K}} \sum_{l=1}^{L}\left\{\lambda_{l}^{k} \tilde{\lambda}_{j}^{k}(l)\right\}\right) X_{j}^{\mathrm{S}}=\sum_{j=1}^{n} \hat{\lambda}_{j}^{k} X_{j}^{\mathrm{S}} .
\end{aligned}
$$

Similarly, we have

$$
\tilde{Y}^{k} \leq \sum_{j=1}^{n} \hat{\lambda}_{j}^{k} Y_{j}^{k}, \quad \tilde{Y}^{\mathrm{S}} \leq \sum_{j=1}^{n} \hat{\lambda}_{j}^{k} Y_{j}^{\mathrm{S}} .
$$

Therefore, $(\tilde{X}, \tilde{Y})$ satisfies (11) with $\hat{\lambda}_{j}^{k}$ defined by $(28)$, and $(\tilde{X}, \tilde{Y}) \in T^{*}$.

Proof of Proposition 2. Let $(X, Y)$ satisfy (11) with some vectors $\lambda^{k} \in \mathbb{R}_{+}^{n}, k \in \mathcal{K}$. For all $j \in J$, define $\lambda_{j}^{\min }=\min _{k \in \mathcal{K}} \lambda_{j}^{k}$ and $\lambda_{j}^{\max }=\max _{k \in \mathcal{K}} \lambda_{j}^{k}$. Then $(X, Y)$ satisfies (12) with $\lambda_{j}^{\min }, \lambda_{j}^{\max }, j \in J$, and $\lambda^{k}, k \in \mathcal{K}$. Conversely, let $(X, Y)$ satisfy (12) with some $\lambda_{j}^{\min }, \lambda_{j}^{\max }, j \in \mathcal{J}$, and $\lambda^{k}, k \in \mathcal{K}$. Then (12b) and (12e) imply (11b), and (12d) and (12f) imply (11d).

Proof of Proposition 3. By Theorem 1, technology $T_{\text {MVRS }}$ is defined by conditions (11). Denote $W$ the set of all solutions $\left\langle X, Y, \lambda^{k} \mid \forall k\right\rangle$ that satisfy conditions (11) and the nonnegativity conditions $X, Y \geq 0$. The set $W$ is defined by a finite number of linear inequalities and equalities. Therefore, $W$ is a polyhedral set. Technology $T_{\text {MVRS }}$ is the projection of $W$ on its dimensions $X$ and $Y$. By the projection lemma (see, e.g., its use for the proof of a similar Proposition 1 in Podinovski et al. 2016), $T_{\text {MVRS }}$ is a polyhedral set.

Proof of Proposition 4. The standard VRS technology corresponds to the special case of (11), where we use the same vector of weights $\lambda^{k}=\lambda$ for all component processes $k \in \mathcal{K}$.

Proof of Proposition 5. The fact that Axiom $3^{*}$ implies Axiom 3 is trivial. Conversely, let $T$ satisfy Axioms 3 and $4^{*}$. We need to prove that $T$ satisfies Axiom $3^{*}$. Let $\left(\tilde{X}_{l}, \tilde{Y}_{l}\right) \in T, l=1, \ldots, L$, and let vectors $\lambda^{k} \in \mathbb{R}_{+}^{L}$ be such that $1^{\top} \lambda^{k}=1, k \in \mathcal{K}$. We need to prove that the $\operatorname{DMU}(\tilde{X}, \tilde{Y})$ defined by (10) is in T. Restate (10) as follows: 


$$
\begin{aligned}
& \tilde{X}^{k}=\sum_{l=1}^{L}(1 / L)\left(L \lambda_{l}^{k} \tilde{X}_{l}^{k}\right), \quad \forall k, \\
& \tilde{X}^{\mathrm{S}}=\sum_{l=1}^{L}(1 / L)\left(\max _{k \in \mathcal{K}}\left\{L \lambda_{l}^{k}\right\} \tilde{X}_{l}^{\mathrm{S}}\right), \\
& \tilde{Y}^{k}=\sum_{l=1}^{L}(1 / L)\left(L \lambda_{l}^{k} \tilde{Y}_{l}^{k}\right), \quad \forall k, \\
& \tilde{Y}^{\mathrm{S}}=\sum_{l=1}^{L}(1 / L)\left(\min _{k \in \mathcal{K}}\left\{L \lambda_{l}^{k}\right\} \tilde{Y}_{l}^{\mathrm{S}}\right) .
\end{aligned}
$$

Let $\rho_{l}^{k}=L \lambda_{l}^{k}, \forall k, l$. For each $l=1, \ldots, L$, define the DMU

$$
\left(\begin{array}{c}
\hat{X}_{l} \\
\hat{Y}_{l}
\end{array}\right)=\left(\begin{array}{c}
\rho_{l}^{1} \tilde{X}_{l}^{1}, \ldots, \rho_{l}^{K} \tilde{X}_{l}^{K}, \max _{k \in \mathcal{K}}\left\{\rho_{l}^{k}\right\} \tilde{X}_{l}^{\mathrm{S}} \\
\rho_{l}^{1} \tilde{Y}_{l}^{1}, \ldots, \rho_{l}^{K} \tilde{Y}_{l}^{K}, \min _{k \in \mathcal{K}}\left\{\rho_{l}^{k}\right\} \tilde{Y}_{l}^{\mathrm{S}}
\end{array}\right) .
$$

By Axiom $4^{*},\left(\hat{X}_{l}, \hat{Y}_{l}\right) \in T, \forall l$. By Axiom $3, T$ is a convex set. Because $\sum_{l=1}^{L}(1 / L)=1$, the DMU $(\tilde{X}, \tilde{Y})$ defined by $(29)$ or, equivalently, by $(10)$ is in $T$. Therefore, $T$ satisfies Axiom $3^{*}$.

Proof of Theorem 2. Denote $T_{\mathrm{MCRS}}^{*}$ the set of all DMUs $(X, Y) \geq 0$ that satisfy (14) with some vectors $\lambda^{k} \in \mathbb{R}^{n}, k \in \mathcal{K}$. We need to prove that $T_{\mathrm{MCRS}}=T_{\mathrm{MCRS}}^{*}$. The proof follows from Lemmas 2 and 3 proved below. By Lemma 2, $T_{\mathrm{MCRS}}^{*} \subseteq T_{\mathrm{MCRS}}$. By Lemma $3, T_{\mathrm{MCRS}}^{*}$ satisfies Axioms 1,2 , $3^{*}$ and $4^{*}$. Because, by definition, $T_{\mathrm{MCRS}}$ is the intersection of all technologies that satisfy these axioms, we have $T_{\mathrm{MCRS}} \subseteq T_{\mathrm{MCRS}}^{*}$. Therefore, $T_{\mathrm{MCRS}}=T_{\mathrm{MCRS}}^{*}$.

LEMMA 2. $T_{\mathrm{MCRS}}^{*} \subseteq T_{\mathrm{MCRS}}$, where technology $T_{\mathrm{MCRS}}^{*}$ is as defined in the proof of Theorem 2 .

Proof of Lemma 2. Let $(X, Y) \in T_{\mathrm{MCRS}}^{*}$. Then $(X, Y)$ satisfies (14) with some some $\lambda^{k} \in \mathbb{R}^{n}$, $k \in \mathcal{K}$. Repeating the proof of Proposition 5, restate the left-hand side of (14) as (29), where $l$ and $L$ are replaced by $j$ and $n$, respectively. Because $T_{\text {MCRS }}$ satisfies Axioms 1,3 (or $3^{*}$ ) and $4^{*}$, the $\operatorname{DMU}(\tilde{X}, \tilde{Y})$ on the left-hand side of inequalities (14a)-(14d) (restated as $(29))$ is in $T_{\text {MCRS }}$. By Axiom 2, the DMU $(X, Y)$, which appears on the right-hand side of $(14)$, is in $T_{\text {MVRS }}$.

Lemma 3. Technology $T_{\mathrm{MCRS}}^{*}$ defined in the proof of Theorem 2 satisfies Axioms 1, 2, 3* and $4^{*}$.

Proof of Lemma 3. The proof that $T_{\mathrm{MCRS}}^{*}$ satisfies Axioms 1, 2 and $3^{*}$ is similar to the proof of Lemma 1. To prove that $T_{\mathrm{MCRS}}^{*}$ satisfies Axiom $4^{*}$, let $\operatorname{DMU}(X, Y) \in T_{\mathrm{MCRS}}^{*}$ and let $\rho \in \mathbb{R}_{+}^{K}$. We need to prove that DMU $(X(\rho), Y(\rho))$ defined by (13) is in $T_{\mathrm{MCRS}}^{*}$. 
$\operatorname{DMU}(X, Y)$ satisfies (14) with some vectors $\lambda^{k}, k \in \mathcal{K}$. Define $\hat{\lambda}_{j}^{k}=\rho^{k} \lambda_{j}^{k}$, for all $j \in J, k \in \mathcal{K}$. Then, from (14a), we have

$$
\sum_{j=1}^{n} \hat{\lambda}_{j}^{k} X_{j}^{k}=\rho^{k} \sum_{j=1}^{n} \lambda_{j}^{k} X_{j}^{k} \leq \rho^{k} X^{k}=X(\rho)^{k}, \forall k .
$$

Furthermore, from from (14b), we have

$$
\begin{aligned}
\sum_{j=1}^{n} \max _{k \in \mathcal{K}}\left\{\hat{\lambda}_{j}^{k}\right\} X_{j}^{\mathrm{S}} & =\sum_{j=1}^{n} \max _{k \in \mathcal{K}}\left\{\rho^{k} \lambda_{j}^{k}\right\} X_{j}^{\mathrm{S}} \\
& \leq \sum_{j=1}^{n} \max _{k \in \mathcal{K}}\left\{\rho^{k}\right\} \max _{k \in \mathcal{K}}\left\{\lambda_{j}^{k}\right\} X_{j}^{\mathrm{S}} \leq \rho^{\max } X^{\mathrm{S}}=X(\rho)^{\mathrm{S}}
\end{aligned}
$$

Similarly, from (14c) and (14d), we have

$$
\sum_{j=1}^{n} \hat{\lambda}_{j}^{k} Y_{j}^{k} \geq Y(\rho)^{k}, \forall k ; \quad \sum_{j=1}^{n} \max _{k \in \mathcal{K}}\left\{\hat{\lambda}_{j}^{k}\right\} Y_{j}^{\mathrm{S}} \geq Y(\rho)^{\mathrm{S}}
$$

Therefore, DMU $(X(\rho), Y(\rho))$ satisfies (14) with vectors $\hat{\lambda}^{k}, k \in \mathcal{K}$, and is in $T_{\mathrm{MCRS}}^{*}$. Therefore, $T_{\text {MCRS }}^{*}$ satisfies Axiom $4^{*}$.

Proofs of Propositions 6-8. The proofs are similar to the proofs of Propositions 2-4, and are not given.

Proof of Theorem 3. We first declare $\lambda^{\max }$ and $\lambda^{\text {min }}$ sign-free vectors. This does not affect the optimal solution $\eta^{*}$. Let $v \in \mathbb{R}_{+}^{m}$ and $u \in \mathbb{R}_{+}^{s}$ be dual to constraints (16b) and (16c), and (16d) and (16e), respectively. Let $\xi_{j}^{k} \geq 0$ and $\zeta_{j}^{k} \geq 0$ be dual to (16e) and (16f), for all $j \in J$ and $k \in \mathcal{K}$, respectively. Let $\omega^{k}$ be dual to $(16 \mathrm{~h}), k \in \mathcal{K}$. Then the dual to (16) is stated as follows:

$$
\begin{aligned}
& \min \quad v^{\top} X_{o}+\sum_{k=1}^{K} \omega^{k} \\
& \text { s.t. } \quad u^{\top} Y_{o}=1, \\
& \sum_{i \in \mathrm{I}^{k}} v_{i} X_{j i}-\sum_{r \in \mathrm{O}^{k}} u_{r} Y_{j r}+\xi_{j}^{k}-\zeta_{j}^{k}+\omega^{k} \geq 0, \quad \forall j, k \\
& \sum_{i \in \mathrm{I}^{\mathrm{S}}} v_{i} X_{j i}-\sum_{k=1}^{K} \xi_{j}^{k}=0, \quad \forall j, \quad \forall j, \\
& -\sum_{r \in \mathrm{O}^{\mathrm{S}}} u_{r} Y_{j r}+\sum_{k=1}^{K} \zeta_{j}^{k}=0, \quad \forall j
\end{aligned}
$$




$$
u, v \geq 0, \xi_{j}^{k}, \zeta_{j}^{k} \geq 0, \forall j, k, \omega^{k} \operatorname{sign} \text { free, } \forall k
$$

Program (17) is obtained by the substitution of variables $\xi_{j}^{k}$ and $\zeta_{j}^{k}$ by $p_{j}^{k}$ and $q_{j}^{k}, \forall j, k$ :

$$
\xi_{j}^{k}=p_{j}^{k} \sum_{i \in \mathrm{I}^{\mathrm{S}}} v_{i} X_{j i}^{\mathrm{S}}, \quad \zeta_{j}^{k}=q_{j}^{k} \sum_{r \in \mathrm{O}^{\mathrm{S}}} u_{r} Y_{j r}^{\mathrm{S}} .
$$

Note that constraints (30d) and (30e) become (16d).

Proof of Proposition 9. To be specific, let $\mathrm{I}^{k}=\varnothing, \forall k \in \mathcal{K}$. Let $\operatorname{DMU}(X, Y) \in T_{\mathrm{MCRS}}$. Then inequalities (14a) are omitted and $(X, Y)$ satisfies $(14 \mathrm{~b})-(14 \mathrm{~d})$ with some vectors $\lambda^{k} \in \mathbb{R}_{+}^{n}, k \in \mathcal{K}$. Define $\hat{\lambda}_{j}=\max _{k \in \mathcal{K}}\left\{\lambda_{j}^{k}\right\}, \forall j \in J$. Then (14b)-(14d) imply $\sum_{j=1}^{n} \hat{\lambda}_{j} X_{j}^{\mathrm{S}} \leq X^{\mathrm{S}}, \sum_{j=1}^{n} \hat{\lambda}_{j} Y_{j}^{k} \geq Y^{k}$ and $\sum_{j=1}^{n} \hat{\lambda}_{j} Y_{j}^{\mathrm{S}} \geq Y^{\mathrm{S}}, \forall k \in \mathcal{K}$. Therefore, $(X, Y) \in T_{\mathrm{CRS}}$ and $T_{\mathrm{MCRS}} \subseteq T_{\mathrm{CRS}}$. Because $T_{\mathrm{CRS}} \subseteq T_{\mathrm{MCRS}}$, we have $T_{\mathrm{MCRS}}=T_{\mathrm{CRS}}$. In the case $\mathrm{I}^{k}=\varnothing, k \in \mathcal{K}$, we define $\hat{\lambda}_{j}=\min _{k \in \mathcal{K}}\left\{\lambda_{j}^{k}\right\}, \forall j \in J$. The rest of the proof is similar and is not given. Note that this proof is not valid in the case of technology $T_{\mathrm{MVRS}}$ because $1^{\top} \hat{\lambda}$ is generally not equal to 1 .

Proof of Proposition 10. In the case of VRS, it suffices to prove that $T_{\mathrm{MVRS}} \subseteq T_{\mathrm{VRS}}$. Because $\mathrm{I}^{k}=\mathrm{O}^{k}=\varnothing, \forall k \in \mathcal{K}$, conditions (11a) and (11c) in (11) are omitted. Let DMU $(X, Y) \in T_{\mathrm{MVRS}}$. Then $(X, Y)$ satisfies (11b), (11d)-(11f) with some vectors $\lambda^{k} \in \mathbb{R}_{+}^{n}, \forall k \in \mathcal{K}$. Define $\hat{\lambda}_{j}=\lambda_{j}^{1}, \forall j \in J$. Then (14b), (14d) and (11e) imply $\sum_{j=1}^{n} \hat{\lambda}_{j} X_{j}^{\mathrm{S}} \leq X^{\mathrm{S}}, \sum_{j=1}^{n} \hat{\lambda}_{j} Y_{j}^{\mathrm{S}} \geq Y^{\mathrm{S}}$ and $1^{\top} \hat{\lambda}=1$. Therefore, $(X, Y) \in T_{\mathrm{VRS}}$. The proof that $T_{\mathrm{MCRS}}=T_{\mathrm{CRS}}$ is similar and is not given. 


\section{References}

Afriat SN (1972) Efficiency estimation of production functions. Internat. Econom. Rev. 13(3):568-598.

Banker RD (1984) Estimating most productive scale size using data envelopment analysis. Eur. J. Oper. Res. 17(1):35-44.

Banker RD, Charnes A, Cooper WW (1984) Some models for estimating technical and scale inefficiencies in data envelopment analysis. Management Sci. 30(9):1078-1092.

Banker RD, Thrall RM (1992) Estimation of returns to scale using data envelopment analysis. Eur. J. Oper. Res. 62(1):74-84.

Beasley JE (1995) Determining teaching and research efficiencies. J. Oper. Res. Soc. 46(4):441-452.

Chambers RG, Chung Y, Färe R (1998) Profit, directional distance functions, and Nerlovian efficiency. J. Optim. Theory Appl. 98(2):351-364.

Chambers RG, Färe R (2008) A "calculus" for data envelopment analysis. J. Productivity Anal. 30(3):169175.

Charnes A, Cooper WW, Rhodes E (1978) Measuring the efficiency of decision making units. Eur. J. Oper. Res. 2(6):429-444.

Cherchye L, De Rock B, Dierynck B, Roodhooft F, Sabbe J (2013) Opening the "black box" of efficiency measurement: Input allocation in multioutput settings. Oper. Res. 61(5):1148-1165.

Cherchye L, De Rock B, Walheer B (2016) Multi-output profit efficiency and directional distance functions. Omega 61:100-109.

Cook WD, Green RH (2004) Multicomponent efficiency measurement and core business identification in multiplant firms: A DEA model. Eur. J. Oper. Res. 157(3):540-551.

Cook WD, Hababou M (2001) Sales performance measurment in bank branches. Omega 29(4):299-307.

Cook WD, Hababou M, Tuenter HJH (2000) Multicomponent efficiency measurement and shared inputs in data envelopment analysis: An application to sales and service performance in bank branches. $J$. Productivity Anal. 14(3):209-224.

Cook WD, Harrison J, Imanirad R, Rouse P, Zhu J (2013) Data envelopment analysis with nonhomogeneous DMUs. Oper. Res. 61(3):666-676. 
Podinovski: Technologies with Multiple Component Processes

Article submitted to Operations Research; manuscript no. (Please, provide the mansucript number!)

Cook WD, Zhu J (2006) Incorporating multiprocess performance standards into the DEA framework. Oper. Res. 54(4):656-665.

Cook WD, Zhu J (2011) Multiple variable proportionality in data envelopment analysis. Oper. Res. 59(4):1024-1032.

Cooper WW, Seiford LM, Tone K (2007) Data Envelopment Analysis. A Comprehensive Text with Models, Applications, References and DEA-Solver Software, 2nd ed. (Springer, New York).

Dakpo KH, Jeanneaux P, Latruffe L. (2016) Modelling pollution-generating technologies in performance benchmarking: Recent developments, limits and future prospects in the nonparametric framework. Eur. J. Oper. Res. 250(2):347-359.

Ding J, Feng C, Bi G, Liang L, Khan MR (2015) Cone ratio models with shared resources and nontransparent allocation parameters in network DEA. J. Productivity Anal. 44(2):137-155.

Dyson RG, Allen R, Camanho AS, Podinovski VV, Sarrico CS, Shale EA (2001) Pitfalls and protocols in DEA. Eur. J. Oper. Res. 132(2):245-259.

Färe R, Grosskopf S, Logan J (1983) The relative efficiency of Illinois electric utilities. Resources and Energy $5(4): 349-367$.

Färe R, Grosskopf S, Lovell CAK (1985) The Measurement of Efficiency of Production (Kluwer Academic Publishers, Boston).

Färe R, Grosskopf S, Lovell CAK (1994) Production Frontiers (Cambridge University Press, Cambridge).

Førsund FR, Hjalmarsson L (2004) Calculating scale elasticity in DEA models. J. Oper. Res. Soc. 55(10):1023-1038.

Frisch R (1965) Theory of production (D. Reidel Publishing Company, Dordrecht).

Fukuyama H (2000) Returns to scale and scale elasticity in data envelopment analysis. Eur. J. Oper. Res. 125(1):93-112.

Hadjicostas P, Soteriou AC (2006) One-sided elasticities and technical efficiency in multi-output production: A theoretical framework. Eur. J. Oper. Res. 168(2):425-449.

Imanirad R, Cook WD, Zhu J (2013) Partial input to output impacts in DEA: Production considerations and resource sharing among business subunits. Naval Res. Logist. 60(3):190-207. 
Kao C (2014) Network data envelopment analysis: A review. Eur. J. Oper. Res. 239(1):1-16.

Pham MD, Zelenyuk V (2019) Weak disposability in nonparametric production analysis: A new taxonomy of reference technology sets. Eur. J. Oper. Res. 274(1):186-198.

Podinovski VV (2017) Returns to scale in convex production technologies. Eur. J. Oper. Res. 258(3):970-982.

Podinovski VV, Bouzdine-Chameeva T (2019) Cone extensions of polyhedral production technologies. Eur. J. Oper. Res. 276(2):736-743.

Podinovski VV, Bouzdine-Chameeva T (2020) Consistency of returns-to-scale characterizations of production frontiers with respect to model specification. Eur. J. Oper. Res. 280(2):609-620.

Podinovski VV, Chambers RG, Atici KB, Deineko ID (2016) Marginal values and returns to scale for nonparametric production frontiers. Oper. Res. 64(1):236-250.

Podinovski VV, Førsund FR (2010) Differential characteristics of efficient frontiers in data envelopment analysis. Oper. Res. 58(6):1743-1754.

Podinovski VV, Olesen OB, Sarrico CS (2018) Nonparametric production technologies with multiple component processes. Oper. Res. 66(1):282-300.

Ray SC (2004) Data envelopment analysis. Theory and techniques for economics and operations research (Cambridge University Press, Cambridge).

Sahoo BK, Tone K (2015) Scale elasticity in non-parametric DEA approach. Zhu J, ed. Data Envelopment Analysis: A Handbook of Models and Methods (Springer Science + Busines Media, New York) 269-290.

Shephard RW (1974) Indirect Production Functions, Mathematical Systems in Economics No. 10 (Anton Hain, Meisenheim am Glan).

Thanassoulis E, Kortelainen M, Johnes G, Johnes J (2011) Costs and efficiency of higher education institutions in England: A DEA analysis. J. Oper. Res. Soc. 62(7):1282-1297.

Walheer B (2018) Disaggregation of the cost Malmquist productivity index with joint and output-specific inputs. Omega 75:1-12.

Zelenyuk V (2013) A scale elasticity measure for directional distance function and its dual: Theory and DEA estimation. Eur. J. Oper. Res. 228(3):592-600. 